\author{
RYERSON UNIVERSITY \\ FALCULTY OF ENGINEERING, ARCHITECHTURE AND SCIENCE \\ DEPARMENT OF AEROSPACE ENGINEERING
}

\title{
Bio Modelling for Comfort Aircraft Chair Design
}

Xianzhi Zhong

AER870 Aerospace Engineering Thesis - Final Report

Faculty Advisor: Dr. Fengfeng (Jeff) Xi

Date: April 2020 


\section{Acknowledgements}

My deep gratitude goes firstly to my thesis supervisor Dr. Fengfeng (Jeff) Xi for providing me the great opportunity to participate and work on the interesting research. His passion, patient guidance and valuable support kept me constantly engaged with my research and made my time exiting and enjoyable throughout the semester.

My appreciation also extends to my colleagues who helped make this research unforgettable and fruitful to me. Sean Liu's initial guidance and mentoring has been valuable. I also want to thank my family and friends. Their support and encouragement enhanced my confidence and gave me power to face challenges and move forward in my academic life. 


\section{Abstract}

The work presented in this report is to help develop and complete the methodology that can quickly predict the pressure distribution and estimate the comfortability of the aircraft seat. For this thesis, the human back and spine are introduced and modelled as the focus is only on the backrest of the aircraft seat. The bio modelling of the back and spine consists of the geometry including the spine curvature and back shape at various conditions. The variables include the body type of the sitter, the sitting posture and the backrest recline angle. Multiple cases of the body condition combining the these mentioned variables were modelled, which generates a comparatively inclusive human model for the future work of pressure distribution analysis. The initial building of the spine curve is based on an existing spine data, and the back shape is captured by experiments using 3D scanning technology. Forces acting on the spine are also obtained as a part of the modelling.

With this more complete the inclusive bio model of the body, the pattern of the contact and pressure can then determine a more efficient configuration of cushion or aircraft seat innovation and design to achieve a better comfort. 


\section{Contents}

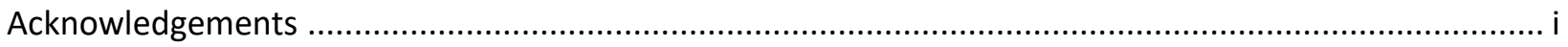

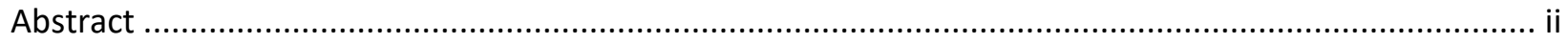

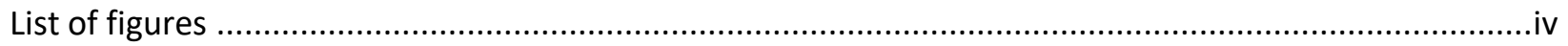

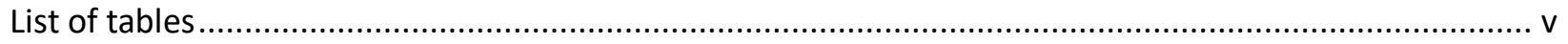

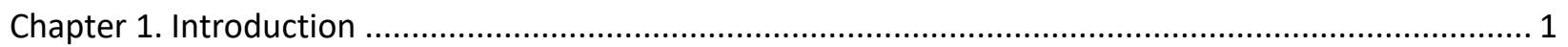

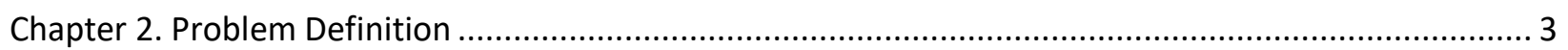

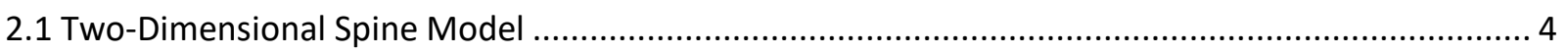

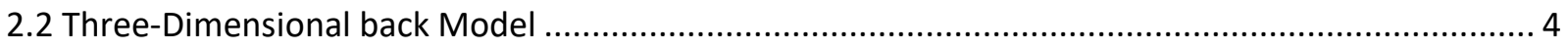

Chapter 3. Modelling and Force Analysis ........................................................................................ 7

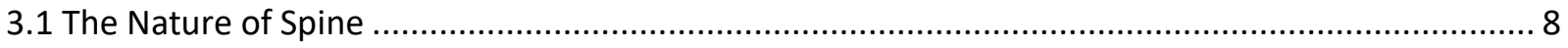

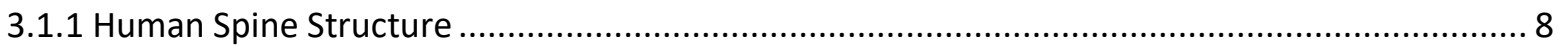

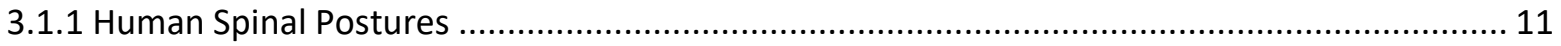

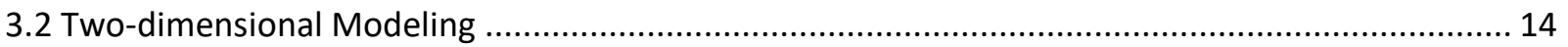

3.2.1 Theory - Brief Overview of Polynomial Curve Fitting - Least squares Approach ..................... 14

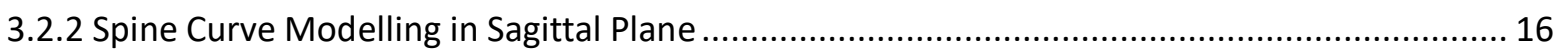

3.2.3 Spine force modelling in sagittal plane ....................................................................... 21

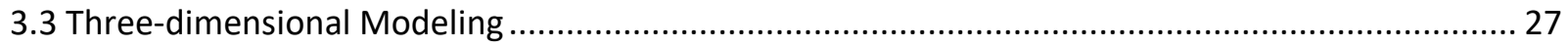

3.3.1 Spine Curve Modeling in a Three-dimensional Space ....................................................... 27

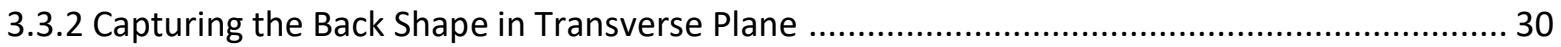

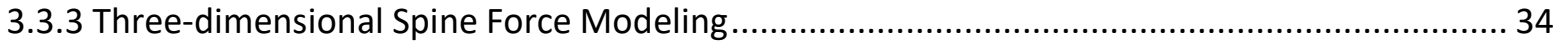

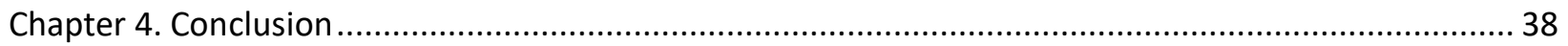

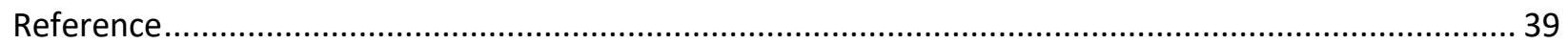

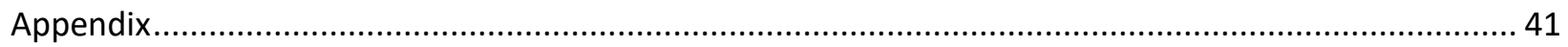




\section{List of figures}

Figure 1. Planes of the body [11]

Figure 2. Posture of side leaning [12]

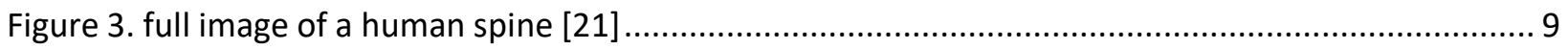

Figure 4. The insight of a vertebra seen from above and from the right side [14] ................................ 10

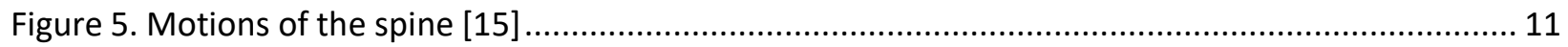

Figure 6. The available 3-mode motion range of all spinal joints [16] ................................................. 12

Figure 7. A typical load-displacement curve for a spinal ligament or motion segment. The physiologic range of motion is composed of the neutral zone (NZ), the elastic zone (EZ) and the plastic zone (PZ) [17]

Figure 8. The plots of three different postures based on the data collected by Kitazaki and Griffin's data 17

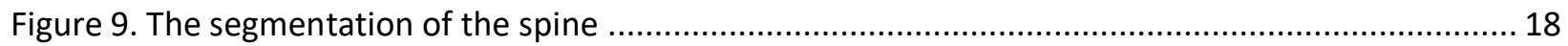

Figure 10. Simplified spine model (lumbar thoracic and cervical region) in sagittal plane by MATLAB. 20

Figure 11. The definition of the turning angles at each joint on the simplified spine model .................... 20

Figure 13. Free body diagram in sagittal plane for one segment that has contact with the seat cushion 21

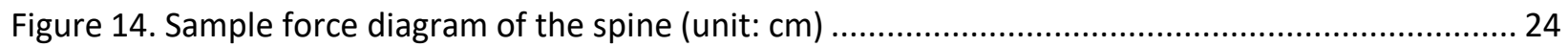

Figure 15. Picture of a sitting human and illustration of backrest recline angle $(\varphi)$............................. 25

Figure 16. The calculated thoracic I normal force magnitude vs. seat backrest recline angle .................. 26

Figure 17. The calculated thoracic II normal force magnitude vs. seat backrest recline angle ................ 26

Figure 18. The calculated lumbar normal force magnitude vs. seat backrest recline angle ..................... 27

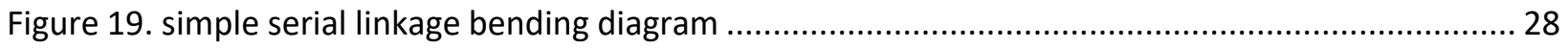

Figure 20. The segmented spine with two levels of lateral bending (half neutral zone "slight bending" and neutral zone maximum bending) on coronal plane (unit: $\mathrm{cm}$ ) (a) with the erect posture on sagittal plane (b) with the normal posture on sagittal plane (c) with the slouched posture on sagittal plane...... 29 Figure 21. The three-dimensional segmented spine model with two levels of lateral bending (half neutral zone "slight bending" shown in red and neutral zone maximum bending shown in blue) when the backrest angle is 90 degrees (unit: $\mathrm{cm}$ ) (a) with the erect posture on sagittal plane (b) with the normal posture on sagittal plane $(\mathrm{c})$ with the slouched posture on sagittal plane ..................................3 30 Figure 22. the illustration of obtaining the back shape on transverse plane ........................................ 31 Figure 23. Collection for the 3D scan figure for each subject. (a) subject 1 with a BMI of 18.8 ; (b) subject 2 with a BMI of 20.1; (c) subject 3 with a BMI of 22.9; (d) subject 4 with a BMI of 29.1; (e) subject 5 with a BMI of 31.9

Figure 24. Free body diagram in the coronal plane for one segment that has contact with the seat

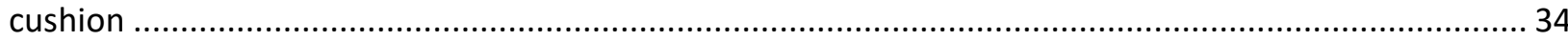

Figure 25. the force diagram of the spine when the backrest recline angle is 105 degree, 120 degree and 135 degree and the spine bends laterally the most within the neutral zone (axis unit: $\mathrm{cm}$ ) (a) erect

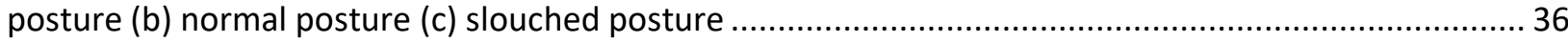
Figure 26. The calculated thoracic I total force magnitude at the contact point vs. seat backrest recline angle

Figure 27. The calculated thoracic II total force magnitude at the contact point vs. seat backrest recline angle 
Figure 28. The calculated lumbar total force magnitude at the contact point vs. seat backrest recline

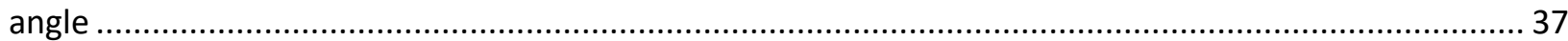

Figure 29. Polynomial fitting curve for a spine with erect posture .................................................... 42

Figure 30. Polynomial fitting curve for a spine with normal posture ................................................. 42

Figure 31. Polynomial fitting curve for a spine with slouched posture ............................................... 43

Figure 32. The back shape on the transverse plane of the body at the different level of lumbar, thoracic I and thoracic II. (a) thoracic I of subject 1 with BMI of 18.8 (b) thoracic II of subject 1 with BMI of 18.8 (c) lumbar of subject 1 with BMI of 18.8 (d) thoracic I of subject 2 with BMI of 20.1 (e) thoracic II of subject 2 with BMI of 20.1 (f) lumbar of subject 2 with BMI of 20.1 (g) thoracic I of subject 3 with BMI of 22.9 (h) thoracic II of subject 3 with BMI of 22.9 (i) lumbar of subject 3 with BMI of 22.9 (j) thoracic I of subject 4 with BMI of 29.1 (k) thoracic II of subject 4 with BMI of 29.1 (I) lumbar I of subject 4 with BMI of $29.1(\mathrm{~m})$ thoracic I of subject 5 with BMI of 31.9 (n) thoracic II of subject 5 with BMI of 31.9 (o) lumbar of subject 5 with BMI of 31.9.

Figure 33. the force diagram of the erected spine when the backrest recline angle is 105 degree, 120 degree and 135 degree

Figure 34. the force diagram of the normal spine when the backrest recline angle is 105 degree, 120 degree and 135 degree 47

Figure 35. the force diagram of the slouched spine when the backrest recline angle is 105 degree, 120 degree and 135 degree

\section{List of tables}

Table 1. Lufthansa's seat dimensions for economy and business class [6] ........................................... 2

Table 2. The standard weight status categories associated with BMI ranges for adults [13] ................... 6

Table 3. the collection of vertebrae of the human spine ................................................................. 9

Table 4. Average neutral zones for rotatory main motions of the spinal motion segments [16] ............. 13

Table 6. Spine segments and their length ratio to the full spine...................................................... 19

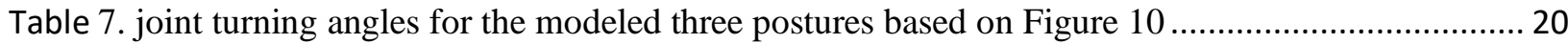

Table 8. head mass and segmental mass of the spine for cases with BMI of $25,30,35,40 \ldots \ldots \ldots \ldots \ldots \ldots . . . . . . .23$

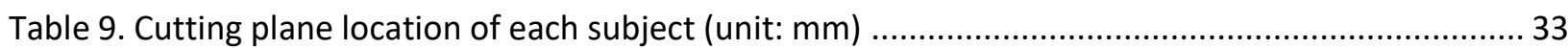

Table 11. Node locations for the head mass, spinal beams and pelvic mass in the three postures .......... 41 


\section{Chapter 1. Introduction}

As traveling by airplane is usual with the increasing interactions between different regions around the world, people's expectation is not only transporting from one place to another but also the experience onboard. The comfort on the aircraft becomes so important that it has been a factor that affects people's choice of transportation, especially for a long-distance flight as the passenger is limited in relatively small cabin area. A leading market analysis company, Frost \& Sullivan also reported that the passenger comfort has been one key driver of the aircraft cabin reconfiguration [1]. During the journey onboard, passengers are seating in the aircraft seat, breathing the air that comes from the air conditioning system, sensing the environmental temperature and cabin pressure, etc. no matter it is the case of commercial or private business jet. Based on the comfort study done by Vink and Brauer [2], 'Pressure and Touch' is one key input to comfort, i.e. the pressure between the seat or handle and the human body sensed by the human skin is of the factors that determines how comfortable people feel. The overall comfort is not easy to achieve, since comfort is usually a subjective feeling, and the results can then vary from people to people. However, Looze, Kuijt-Evers, and Dieënfor [3] found that the pressure distribution is the objective measure with the most clear association with the subjective ratings regarding the sitting comfort. Jiang's research team [4] also indicates that a competitive comfort seat design must be based on detailed pressure analysis, which is the evaluation indicator for sitting comfort. In other words, the comfortability of the seat may be objectively determined if the pressure distribution is obtainable. In order to achieve a good pressure distribution that maximizes the sitting comfort, various innovative designs and ideas were initiated, such as the concept of Chair with Synchronized Tilting Seat and Back, which was published as a patent in 2002 [5] The seat innovation for achieving a better comfort has to respect the allowable design space, as the cabin volume is always limited and the aircraft tends to equip as many seats for economic benefits. One example spacing data for the seat and each passenger is collected in Table 1. 
Table 1. Lufthansa's seat dimensions for economy and business class [6]

\begin{tabular}{|c|c|c|}
\hline & Economy Class & Business Class \\
\hline Seat pitch & 31 inches & 64 inches \\
\hline Seat shoulder width & 17 inches $\sim 18$ inches & 26.4 inches \\
\hline
\end{tabular}

Seat cushion is also related to the sitting comfort. As noted by Kolish and Taboun [7], one requirement of a good seat cushion is to produce a more evenly distributed pressure around the ischial tuberosities where the pressure peaks. Many researches about comfort testing or evaluation treat cushion selection as a variable. For instance, the cushion foam's characteristic was found to be a significant factor contributing to subjective factor in the study done by Vincent, Bhise and Mallick [8] about comfort prediction. Cushion is what contacts directly with the human body, and the studies about the pressure at sitting condition demonstrates that the seat cushion plays a very important role as for the sitting comfort.

In conclusion, an ideal pressure distribution between the cushion surface and the human body (achieved by e.g. innovating seat design or changing the cushion) is the objective reference that measures the comfortability of a seat. The current available pressure evaluation methods of empirical approach and Finite Element Method (FEM) face multiple limitations. Empirical measurement using expensive equipment requires large investment on time and resources for prototyping and field testing. FEM uses computer aided engineering technology to simulate the pressure, which is cheaper compared to the empirical method. However, FEM requires realistic computer models for the seated human, the seat, and seat-human interactions to do the calculation [9], and the available qualified models are very limited. Therefore, a method for the pressure prediction by investigating the interaction between the human back and aircraft seat backrest based on various body types and human postures was initiated by Liu [10], which can achieve an improved efficiency at reduced cost. In Liu's method, a bio modeling for the sitter is required, and a more complete human modeling developed would make the method capable of investigating more cases of the pressure distribution while the sitter is at various postures while sitting on the aircraft seat. 


\section{Chapter 2. Problem Definition}

The research objective is to develop an human modelling, based on which the contact pressure simulation method developed by Liu [10] can be applied more inclusively with more sitting cases. The sitting cases include the sitting with various postures, backrest reline angle and sitter's Body Mass Index (BMI). The inclusive method will then better study the interaction between the human body and the aircraft seat with the application of bio mechanics and contact mechanics. As a result, it is expected to simulate the pressure distribution for more cases according to the bio modeling that will be presented in this report. The simulated pressure distribution will provide a clear view of the stressed areas on the seat backrest and the evidence of improving or innovating the aircraft seat design to be more comfortable and competitive.

In order to model the human back, the geometry information must be collected. The geometry of the normal human back is assumed to be symmetrical to the central spine while standing and sitting straight. According to the anatomical plane (as shown in Figure 1), the back model can be defined by the twodimensional spine curve (as the guideline) in sagittal plane, and the back shape at different height on the transverse plane. The posture of leaning forward or backward is defined by the spine variation within the sagittal plane; the postures of leaning left or right within coronal plane; body twisting about the center line and the BMI difference of the modelled body are the geometry variation within the Transverse plane.

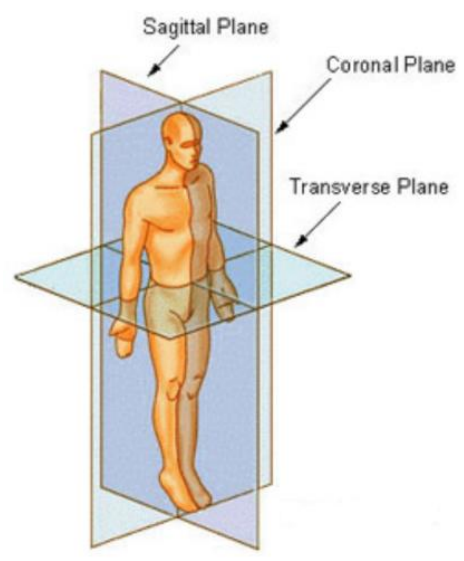

Figure 1. Planes of the body [11] 


\subsection{Two-Dimensional Spine Model}

The two-dimensional spine model is on the sagittal plane of the human body. Only the region of the human back is considered since the comfort study is limited on the seat backrest as presented in this report. On the sagittal plane, the most important figure that can best describe the back geometry is the spine curve. Because the research is to define various conditions that the body may preform (e.g. various postures) spine curve at difference postures are to be obtained.

In order to simulate the pressure distribution, Liu's method [10] requires the a segmented spine model. i.e. the obtained spine curve must be simplified to a serial linkage. The head would be on top of it, and at the bottom would it be the ischial tuberosities. The segmenting process needs to be well explained to show the segmented model is valid in place of the smooth spine curve that is closer to the real case. In order to define a serial linkage (segmented spine model) that is made of the links and joints, the link length and the angle at each joint must be determined in a way that is convenient for the calculations according to computational dynamics. Therefore, through simplifying the spine curve, different postures can be performed by a different combination of the rotation angles at each joint.

The two-dimensional spine modelling is the foundation of the three-dimensional back modeling, which will be discussed in further detail in the following section.

\subsection{Three-Dimensional back Model}

Based on the expectations of the two-dimensional modelling as described in the previous section, a three-dimensional model for the human spine at various conditions is beyond the sagittal plane. Firstly, considering the activities or postures that make body leaning left or right from the center line (as demonstrated in Figure 2), the spine curve defined in the sagittal plane in this case can move within the coronal plane. Even though it is not healthy to sit leaning to one side for long, it cannot be denied that people do choose to sit with this manner intentionally or unintentionally while sleeping for example. 
Therefore, a three-dimensional spine model is required if considering the postures that relates not only the sagittal plane but also the coronal plane, and a three-dimensional model should be generated just to cover the usual scenarios.

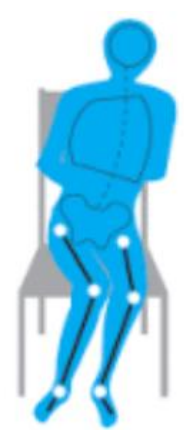

Figure 2. Posture of side leaning [12]

The simplification of the spine curve (segmented spine model) is also used in three-dimensional modeling. But to describe the kinetic on coronal plane (other than sagittal plane), rotation angles at each joint that defines the posture of the human body must become three-dimensional, which means that the joint has to be assumed as a spherical joint ( $\mathrm{S}$ joint) allowing special rotations, instead of a revolute joint (R-joint) as assumed in the two-dimensional cases in sagittal plane.

Sitting posture is not the only variable that affects the pressure distribution. The body type of the sitter should also be considered as one of the impact factors of the pressure distribution on the backrest of the seat. The Body Mass Index (BMI) is a good indicator that can broadly measure the body fatness and categorize the body type of the sitter as BMI is a function of body mass and height $\left(\mathrm{BMI}=\frac{\text { weight }(\mathrm{kg})}{\operatorname{height}(\mathrm{m})^{2}}\right)[13]$. Table 2 collects the multiple BMI ranges and the corresponding body categories for general adults. In terms of the bio modelling for the back of the sitter, the body type (BMI) variation affects mostly on the backsurface pattern on the transverse plane. In short, the different shapes of the back on the transverse plane for different body type need to be determined to generate a conclusive bio modeling for aircraft seat comfort analysis. 
Table 2. The standard weight status categories associated with BMI ranges for adults [13]

\begin{tabular}{|c|c|}
\hline BMI & Weight Status \\
\hline Below 18.5 & Underweight \\
\hline $18.5-24.9$ & Normal or Healthy Weight \\
\hline $25.9-29.9$ & Overweight \\
\hline 30.0 and above & Obese \\
\hline
\end{tabular}

In the scenario of a sitter sitting on the chair, the gravitational force of the body mass would be the force load that acts on the seat while the seat also provides support to different segments of the human body to hold and balance certain amount of the body mass and moment on the body. To study the comfort by simulating the pressure on the seatback, the loading on the contact surface is required. In this report, not only the geometry of the back of the body needs to be modeled, but also the loadings from the body and the contact reaction forces to the body. The force modeling is applied on both two-dimensional and threedimensional bio modelling cases. 


\section{Chapter 3. Modelling and Force Analysis}

This chapter collects the information used for the modelling and in detail explains the method of modeling the general human back. As previously mentioned in the section of Problem definition, note that not only the geometry, but also the forces in different cases will be modeled in order to complete the simulation of the seat backrest pressure distribution in different scenario.

The modelling tool utilized for the study is MATLAB. MATLAB is a high-performance technical computing language and has become a popular and powerful tool to use for computation, modelling, simulation, data analysis, scientific graphics, etc. In this thesis, MATLAB provides an ideal environment for the analytical process and the presentation of the results.

This chapter is outlined with the following subtopics. To begin with, the object of the modeling, the human spine is introduced from the perspective of biomechanics. A good understanding of the human spine's nature is essential for a proper and professional modeling for the study presented in this report. A valid simulation is expected to simulate the realistic pressure results, which of course would require the professional knowledge that tells the facts about the human spine. Plenty of resources were referred to, and an overview of the human spine is provided in the first subsection of this chapter. After the introduction the human spine, the two-dimensional spine model is then generated. At this stage, the two-dimensional spine model is segmented and the force on the spine is analyzed. Finally, the three-dimensional model can be proceeded with the completion of the two-dimensional modelling. The three-dimensional model includes the leaning condition of the body, which generates the deformation of the spine on coronal plane. Then modelled spine on this stage is in a three-dimensional space, it is also segmented like the two-dimensional model, and the force on the spine is analyzed as well. Moreover, the fatness of the body (measured by BMI) would produce certain pattern on the transverse plane; the acquisition of the patterns applies 3D scanning technology. Further details will be explained in the contents of each of the following subsections. 


\subsection{The Nature of Spine}

Spine, also known as vertebral column, is important for human in many ways. The main functions of the spine are to:

1. Protect the spinal cord, nerve roots and several of the body's internal organs.

2. Provide structural support and balance to maintain an upright posture.

3. Enable flexible motion.

Without a spine, due to the lack of support, a person cannot even stand up or control the movements of the body. In this thesis study, the biomechanical importance of spine is emphasized, which is about the structural support and motion control functions. The human spine is an important structure, providing strength and support as well as permitting the body to bend, stretch, rotate and lean. The impact from the nerves, tendons and muscle are not considered for the current level of study presented in this report. Even though the distribution of the muscle and the skin and fat are covering the back and the whole body, the spine geometry defines the pattern of the back to a great extent.

\subsubsection{Human Spine Structure}

The human spine can support the upper body to stand or sit straight. Observing as the front view (the projection on the coronal plane), the spine appears to be rigid and straight for a normal healthy human; observing from the side (the projection on the sagittal plane), the spine forms a slight ' $\mathrm{S}$ ' curve with a kyphotic curve that is convex (curves outward) at the upper and bottom part of the spine (thoracic region), and a lordotic curve that is concave (curves inward) at the lower part of the spine (lumbar region). Figure 3 shows the side view of full human spine and displays the anteroposterior curvatures. A full human spine consists of 33 vertebrae (as displayed in Figure 3) with 24 presacral movable vertebrae that render the spine flexible [14], and is biomechanically separated into 5 regions, which are cervical spine, thoracic spine, 


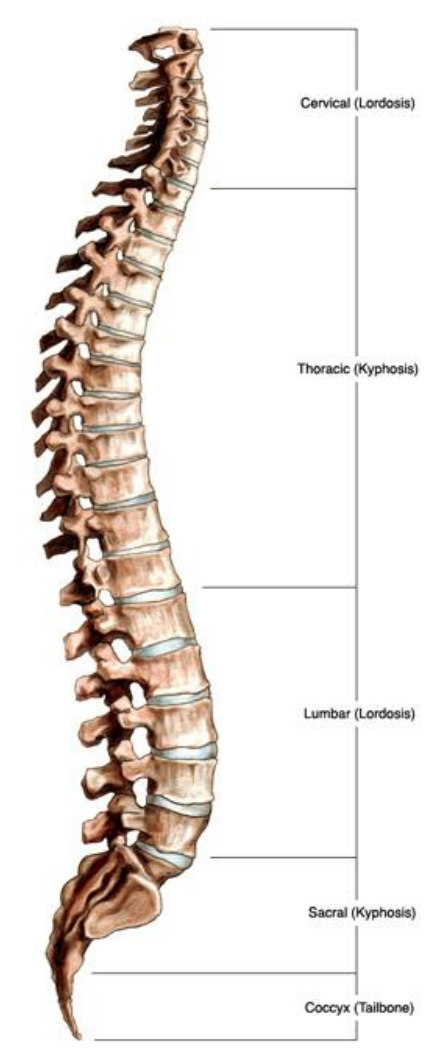

Figure 3. full image of a human spine [21]

lumbar spine, sacral spine and coccyx spine respectively from the top to the bottom. Each of the segments contain certain number of vertebrae; for the convenience of study, each of the vertebra is named by the combination the first letter of the corresponding region's name and the order number. The number of vertebras in each region and the naming for each vertebra is collected in Table 3; the naming order for the mentioned regions are from top to bottom.

Table 3. the collection of vertebrae of the human spine

\begin{tabular}{|c|c|c|}
\hline Spine region & Number of vertebras & Abbreviations of the vertebra \\
\hline Cervical & 7 & $\mathrm{C} 1-\mathrm{C} 7$ \\
\hline Thoracic & 12 & $\mathrm{~T} 1-\mathrm{T} 12$ \\
\hline Lumbar & 5 & $\mathrm{~L} 1-\mathrm{L} 5$ \\
\hline Sacrum & 5 (fused vertebrae) & $\mathrm{S} 1-\mathrm{S} 5$ \\
\hline Coccyx & 4 (fused vertebrae) & $\mathrm{Co} 1-\mathrm{Co} 4$ \\
\hline
\end{tabular}

The motion of the spine is achieved by the relative motion between every two neighbouring presacral vertebras. Figure 4 shows the components of a spinal joint and vertebra and their locations on the 
spinal bone. Each vertebra consists a body and a vertebra arch. The body part is where is pressure is taken if the loading is exerted on the spine. Between every two neighbouring vertebras, there is a connection material called intervertebral disc. The intervertebral disc plays a very important role of bonding different vertebras and provides the cushioning functions performed by the nucleus pulposus inside of the disc when a pressure is loaded. The contact joint between the vertebra also includes the articular, also known as the facet joint. The joint allows the smooth movements between the bone surface, where the cartilage provides an ideal environment of little friction. because of the degree of freedoms provided by the intervertebral disc and the facet joint connection, the spine can bend and rotate many directions with the actions of the muscle. Based on the nature, when doing the three-dimensional modelling of the spine, the joint of the simplified model will be therefore assumed to be a rigid Spherical joint ( $\mathrm{S}$ joint). Note that this assumption is not completely accurate due to the multiple joint connections through the facet joint (sliding motion) and the intervertebral disc (complex compliant volume deformation) between the vertebra. Since the error would be comparatively minor.

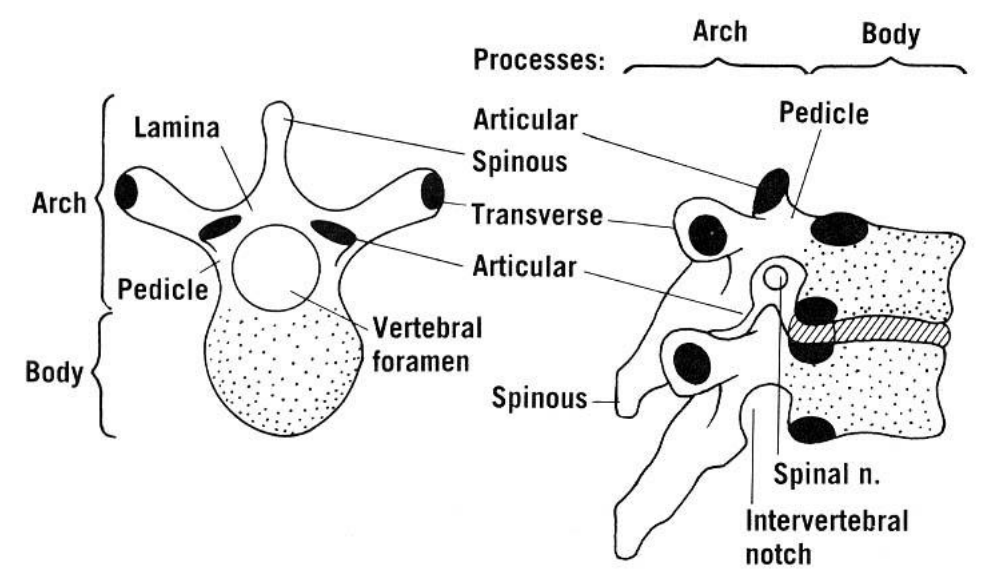

Figure 4. The insight of a vertebra seen from above and from the right side [14] 


\subsubsection{Human Spinal Postures}

Base on the introduction of the spine composition and the description of the spinal joint mechanics from the last section, the deformation of the spine curve is possible with the functioning constrained high degree of freedom joint between the vertebras. Initiated with the action of the core stability muscles (postural muscles), the spine can form extension or flexion in the sagittal plane, lateral bending in the coronal plane and rotation (twisting) in the transverse plane. These modes of motion are as displayed in Figure 5; the angles in the figure just provides a reference line that can better describe the motion. The upper body postures may be formed by the combination of the mentioned spinal motions, which therefore generates many cases of the passenger sitting as for the scenario that is interested in this report.

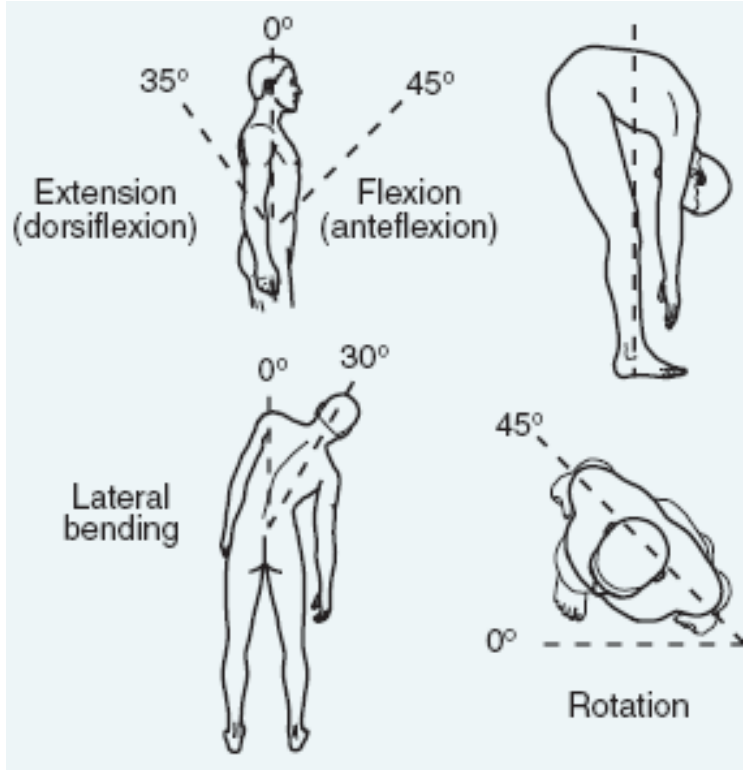

Figure 5. Motions of the spine [15]

The motions within all the body planes have certain ranges. Panjabi and coworkers [16] identified the specific motion range at every joint of the spine. In Figure 6, the standing bar chart for the three modes of motion (Flexion-extension, lateral flexion and rotation) demonstrate the motion range (angle with the unit of degree) for the joints of cervical, thoracic, lumbar and sacrum spinal region. It can be observed that the lumbar region deforms the most when the spine bends in the sagittal plane, while it does not contribute much of the rotation when the spine is twisting in the transverse plane. Comparatively, the lateral motion 
of the spine is more averaged and equal; especially, most vertebras in the thoracic region moves the same amount if the spine flexes laterally.
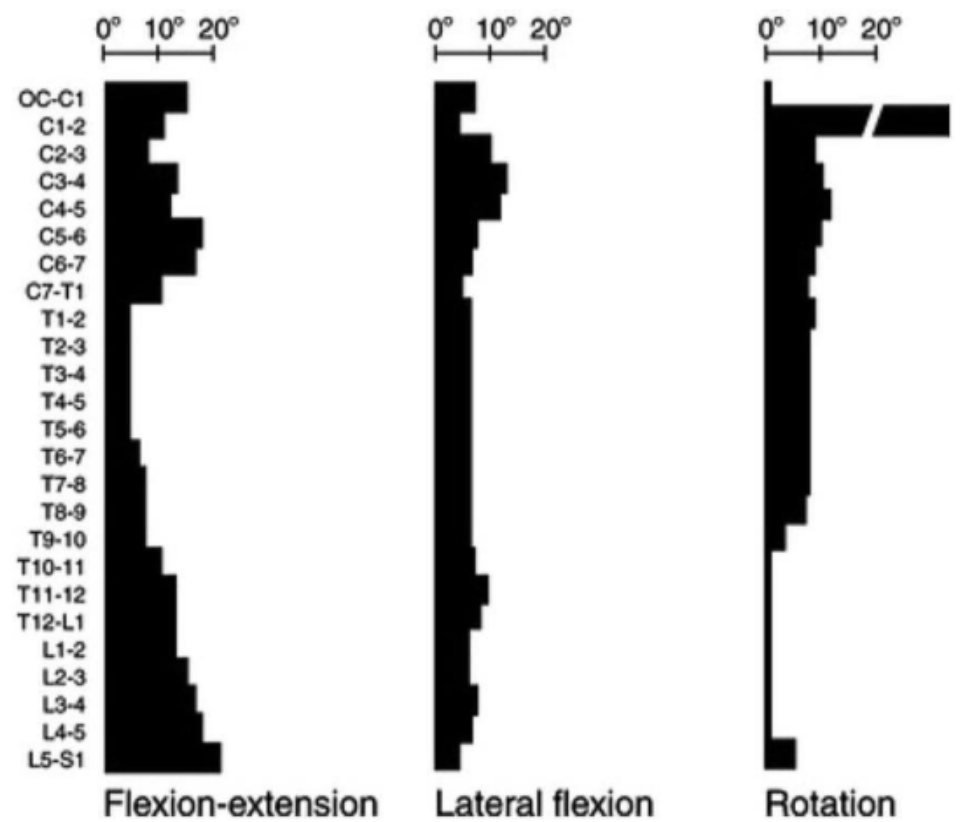

Figure 6. The available 3-mode motion range of all spinal joints [16]

Based on Plaugher and Lopes's study on the spine biomechanics [17], the range of motion is composed of levels of motion. i.e. within the range of motion there are multiple zones categorized by the different physical performance of the spine motion segment while it produces the motion. Figure 7 shows a typical load-displacement curve for spinal ligament or motion segment. Translational movement is expressed in length units and rotational movement is then in degrees. the neutral zone is the first phase of the range of motion, and it is usually a small portion of the range of motion. Motions occurred within the neutral zone will not overcome a lot of tension, and it is reasonably to assume this scenario works for the spine when a person is sitting on the comfortable seat in a relaxed condition. Therefore, the boundary of the neutral zone of the spine segments will be referred to determine the natural lateral bending of the spine. 


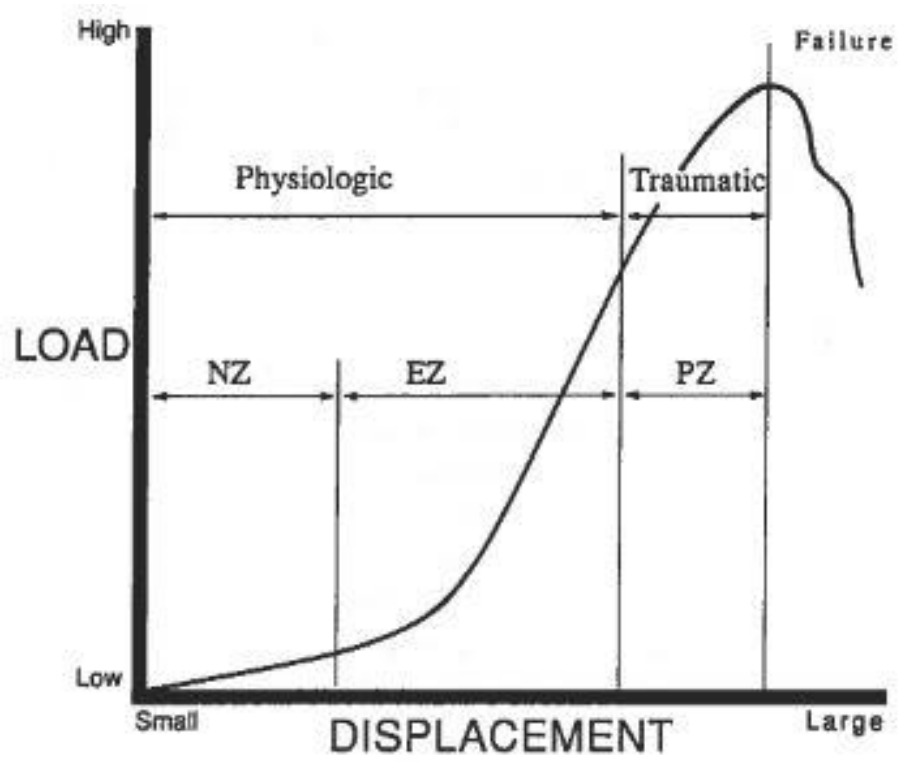

Figure 7. A typical load-displacement curve for a spinal ligament or motion segment. The physiologic range of motion is composed of the neutral zone (NZ), the elastic zone (EZ) and the plastic zone (PZ) [17]

Table 4 shows various neutral zones of the spinal columns, based on which the maximum lateral bending curve of the spine within the neutral range of motion can be determined. This will be discussed in more details in later chapters of spine modelling.

Table 4. Average neutral zones for rotatory main motions of the spinal motion segments [16]

\begin{tabular}{lccc}
\hline \multicolumn{1}{c}{ Region } & Flex/Ext $(\theta \mathrm{X})$ & Lat Bend $(\theta \mathrm{Z})$ & Axial Rot. $(\theta \mathrm{Y})$ \\
\hline C0-C1 & $1.1^{\circ}$ & $1.6^{\circ}$ & $1.5^{\circ}$ \\
C1-C2 & $3.2^{\circ}$ & $1.2^{\circ}$ & $29.6^{\circ}$ \\
C3-C6 & $4.9^{\circ}$ & $4.0^{\circ}$ & $3.8^{\circ}$ \\
C7-T1/T11-T1 & $1.5^{\circ}$ & $2.2^{\circ}$ & $1.2^{\circ}$ \\
L1-L2/L3-L4 & $1.5^{\circ}$ & $1.6^{\circ}$ & $0.7^{\circ}$ \\
L5-S1 & $3.0^{\circ}$ & $1.8^{\circ}$ & $0.4^{\circ}$ \\
\hline
\end{tabular}




\subsection{Two-dimensional Modeling}

\subsubsection{Theory - Brief Overview of Polynomial Curve Fitting - Least squares Approach}

Plotting a fitting curve based on provided data and defining its equation is a quite frequent application in data processing visualization. One method is polynomial curve fitting with the least square error approach. This method is flexible in terms of the goodness of fitting because the degree of the fitted polynomial approximation can be adjusted accordingly. The process of fitting the approximation curve to the given data is simplified and made more efficient with the aid of computer software (MATLAB). The available computing power would be able to generate very fine result that best describe the trend of the data. The calculation theory of the fitting a curve with least square approach is presented as follows.

It is basic to firstly know the general form of a $\mathrm{m}^{\text {th }}$ degree polynomial equation:

$$
f(x)=a_{m} x^{m}+a_{m-1} x^{m-1}+a_{m-2} x^{m-2}+\cdots+a_{1} x^{1}+a_{0}=\sum_{k=1}^{m} a_{k} x^{k}+a_{0}
$$

For each term the coefficient is $a_{i}$, and there are $(m+1)$ coefficients in the general equation (1):

$$
\left[a_{m}, a_{m-1}, a_{m-2}, \ldots, a_{2}, a_{1}, a_{0}\right]
$$

With the known polynomial degree $\mathrm{m}$ ( $\mathrm{m}$ can be selected for the best curve fitting results with the least error/ maximum accuracy), the equation and fitting curve can then be calculated and plotted if the coefficients shown above in matrix (2) are solved.

In a two-dimensional environment, the set of data points (assuming there are $\mathrm{n}$ data points in total) can be expressed with their coordinates as follows:

$$
\left(x_{1}, y_{1}\right),\left(x_{2}, y_{2}\right),\left(x_{3}, y_{3}\right), \ldots,\left(x_{n}, y_{n}\right)
$$


To find the values of the coefficient of each term. The most common method is the least squares approach, whose general expression for the minimized errors is shown below combined with equation (1)

$$
\text { error }=\sum_{i=1}^{n}\left(y_{i}-f\left(x_{i}\right)\right)^{2}=\sum_{i=1}^{n}\left(y_{i}-\left(\sum_{k=1}^{m} a_{k} x_{i}^{k}+a_{0}\right)\right)^{2}
$$

To find the least error from equation (2), the first derivative with respect to each coefficient from matrix(2) is taken and made equal to zero:

$$
\left[\begin{array}{c}
\frac{\text { derror }}{a_{0}} \\
\frac{\text { derror }}{a_{1}} \\
\frac{\text { derror }}{a_{2}} \\
\vdots \\
\frac{\partial e r r o r}{a_{m}}
\end{array}\right]=\left[\begin{array}{c}
-2 \sum_{i=1}^{n}\left(y_{i}-\left(\sum_{k=1}^{m} a_{k} x_{i}^{k}+a_{0}\right)\right) \\
-2 \sum_{i=1}^{n}\left(y_{i}-\left(\sum_{k=1}^{m} a_{k} x_{i}{ }^{k}+a_{0}\right)\right) x^{1} \\
-2 \sum_{i=1}^{n}\left(y_{i}-\left(\sum_{k=1}^{m} a_{k} x_{i}{ }^{k}+a_{0}\right)\right) x^{2} \\
\vdots \\
-2 \sum_{i=1}^{n}\left(y_{i}-\left(\sum_{k=1}^{m} a_{k} x_{i}^{k}+a_{0}\right)\right) x^{m}
\end{array}\right]=\left[\begin{array}{c}
0 \\
0 \\
0 \\
0
\end{array}\right]
$$

The matrix from equation (4) can be equally transformed to the following matrix and all the summation within the matrix are over $i=1, \ldots, n$.

$$
\left[\begin{array}{ccccc}
n & \sum x_{i} & \sum x_{i}{ }^{2} & \cdots & \sum x_{i}{ }^{m} \\
\sum x_{i} & \sum x_{i}{ }^{2} & \sum x_{i}{ }^{3} & \cdots & \sum x_{i}{ }^{m+1} \\
\sum x_{i}{ }^{2} & \sum x_{i}{ }^{3} & \sum x_{i}{ }^{4} & \cdots & \sum x_{i}{ }^{m+2} \\
\vdots & \vdots & \vdots & \ddots & \vdots \\
\sum x_{i}{ }^{m} & \sum x_{i}{ }^{m+1} & \sum x_{i}{ }^{m+2} & \cdots & \sum x_{i}{ }^{2 m}
\end{array}\right]\left[\begin{array}{c}
a_{0} \\
a_{1} \\
a_{2} \\
\vdots \\
a_{m}
\end{array}\right]=\left[\begin{array}{c}
\sum y_{i} \\
\sum x_{i} y_{i} \\
\sum x_{i}{ }^{2} y_{i} \\
\vdots \\
\sum x_{i}{ }^{m} y_{i}
\end{array}\right]
$$

With the given data sets (from point $\left(x_{1}, y_{1}\right)$ to point $\left(x_{n}, y_{n}\right)$ ), the only unknown is the matrix of coefficient $a$ with the size of $(m \times 1)$. Therefore, the solution of equation (5) for the $a$ matrix will present 
the value of the coefficient of every term in the $\mathrm{m}^{\text {th }}$ degree polynomial equation referring to equation (1). With the solved coefficient for each term in equation (1), the fitting curve is obtained.

\subsubsection{Spine Curve Modelling in Sagittal Plane}

As can be imagined, human spine can deform in many ways at different postures or conditions. Many researches analyze the spine by studying certain cases, which focus on certain typical postures for example. Harrison [18] generated his Full-spine Normal Model after modeling each segments of the spine for body structural rehabilitation, postural correction and a serious of spinal health-related applications. To study the human biomechanical responses to whole-body vibration, Kitazaki and Griffin [19] collected the geometry data (see Table 9 in the Appendix) that describes the body spine in a coordinate system when seated, including mass centers of the head, all spinal beams and the pelvic. In their research, by using an anthropometric stand, the spinal geometry data was collected, and can be used to graph three different body postures of being erect, normal and slouched. They are visually shown in figure 1 and figure 2 . The study and modelling presented in this report will utilized the data same spinal data posted by Kitazaki and Griffin [19] of the three different postures as the case study in the two-dimensional spine modelling in the sagittal plane. 


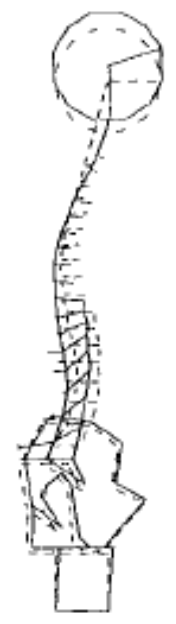

(a)

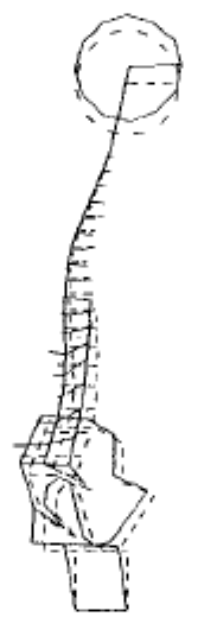

(b)

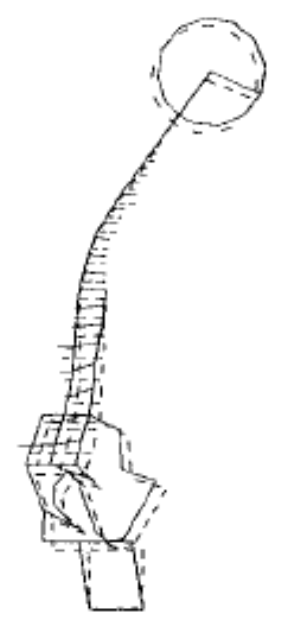

(c)

Figure 8. The plots of three different postures based on the data collected by Kitazaki and Griffin's data

With the available data points, the smooth curve line that describes the trend of the data is fitted with the least square approach of polynomial fitting. For the three postures (erect, normal and slouched), the fitted polynomial curve is presented in Figure 28, Figure 29 and Figure 30 respectively in the Appendix. Based on the data in Table 9, the polynomial fitting equations for all three postures are as follows

$x_{\text {erect }}=f_{\text {erect }}(z)$

$$
\begin{aligned}
& =-1.525 * 10^{-10} z^{7}+6.114 * 10^{-8} z^{6}-9.939 * 10^{-6} z^{5}+8.389 * 10^{-4} z^{4} \\
& -0.03894 z^{3}+0.9667 z^{2}-11.37 z+41.57
\end{aligned}
$$

$x_{\text {normal }}=f_{\text {normal }}(z)$

$$
\begin{aligned}
& =7.239 * 10^{-11} z^{7}-1.621 * 10^{-8} z^{6}-1.05 * 10^{-6} z^{5}+8.818 * 10^{-6} z^{4} \\
& -3.346 * 10^{-3} z^{3}+0.1178 z^{2}-1.245 z-6.512
\end{aligned}
$$

$x_{\text {slouched }}=f_{\text {slouched }}(z)$

$$
\begin{aligned}
& =1.39 * 10^{-10} z^{7}-3.5 * 10^{-8} z^{6}+3.214 * 10^{-6} z^{5}-1.233 * 10^{-4} z^{4} \\
& +1.329 * 10^{-3} z^{3}+0.02428 z^{2}-0.3235 z-10.13
\end{aligned}
$$


With the accurate smooth fitting curve for the spine with different postures, the simplified model can then be obtained. The simplified model requires segmenting the spine curve into sections. As discussed in previous chapter, biomechanically the movable spine is seen as the combination of cervical, thoracic and lumbar region. The segmentation for the spine model also follows the spine regions. Because the thoracic region is longer with 12 vertebras compared with other region, it is divided into 2 regions for the simplified model; they are named thoracic I and thoracic II. Therefore, the segmented simplified spine contains 4 segments (from top to bottom): cervical, thoracic I, thoracic II, lumbar. Figure 9 illustrates the segmentation of the full spine.

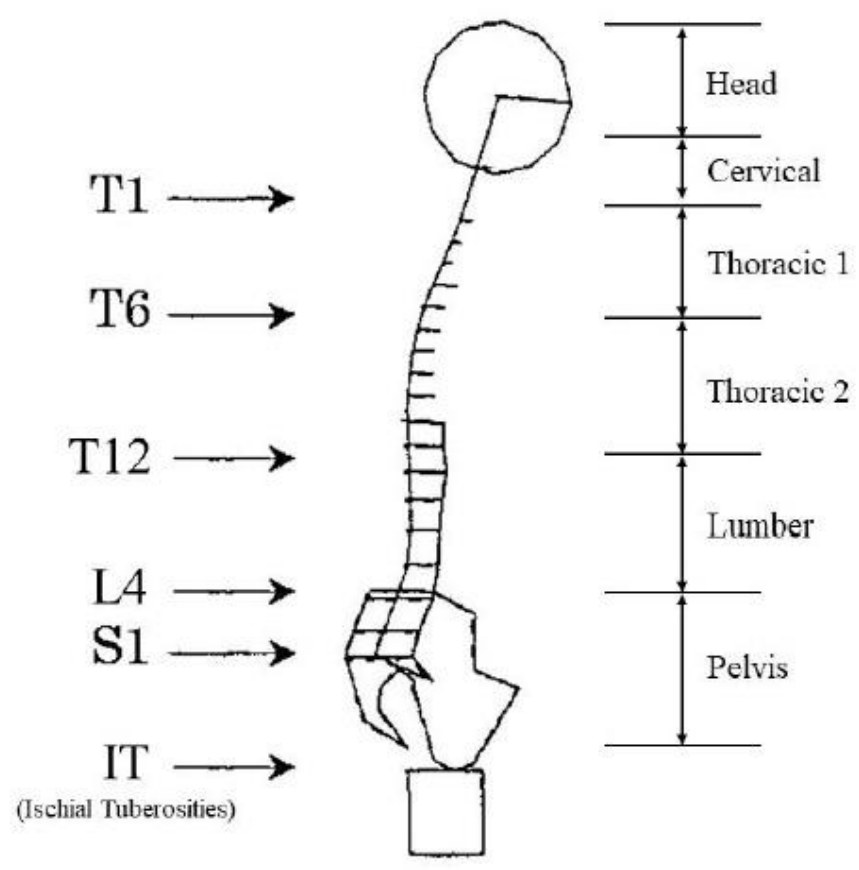

Figure 9. The segmentation of the spine

Based on the fitted curve, the length of each segment and the total length of the spine can also be calculated. The total length of the spine is computed as approximately $0.612 \mathrm{~m}$ for Kitazaki and Griffin's data model [19]. Thus, the percentage ratio of each segment of the spine compared with the full spine length can be found (results are as shown in Table 5) 
Table 5. Spine segments and their length ratio to the full spine

\begin{tabular}{|l|c|}
\hline Segment & Length ratio to the whole spine $\left(\right.$ length ratio $\left.=\frac{\text { segment length }}{\text { spine total length }}\right)$ \\
\hline Cervical region & $21.37 \%$ \\
\hline Thoracic I & $25.64 \%$ \\
\hline Thoracic II & $22.22 \%$ \\
\hline Lumbar & $30.77 \%$ \\
\hline
\end{tabular}

The lengths of the segments are calculated with the arc length equation. With the available function $x=f(z)$ that simulates the spine, the length of it can be easily calculated with the Arc Length Formula

$$
\text { segment length }=\int_{a}^{b} \sqrt{1+\left(\frac{\partial x}{\partial z}\right)^{2}} d z
$$

In this study, $\mathrm{a}$ and $\mathrm{b}$ in the equation above are the $\mathrm{z}$ coordinates of both end points of the segment whose length is to be calculated.

For this provided model, the segmentation does not require finding the arc lengths of the segments because the locations of all the vertebras are available referring to Table 9. However, for later comfort analysis for different subjects, whose spine length vary, finding the length ratio of each segment is necessary. For a different test subject with a different spine length, the segment lengths for this subject can only be found if the length ratio is known, so that the joint locations can be determined. By connecting the located joints can the segmental spinal model be generated. The spine curve is simplified to a simple serial linkage with 4 links and 5 joints for the three postures; they are displayed in Figure 10. To describe the spine model, the angle at each joint is determined. The angles are defined as shown in Figure 11; the values of the joint turning angle for the modeled three postures are collected in Table 6 


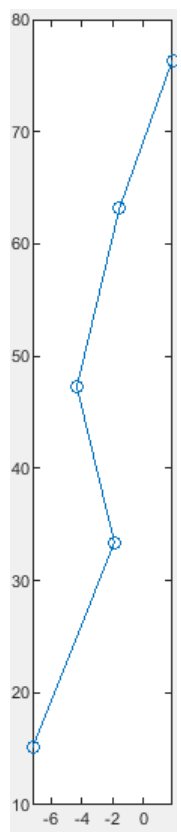

(a) Erect Posture

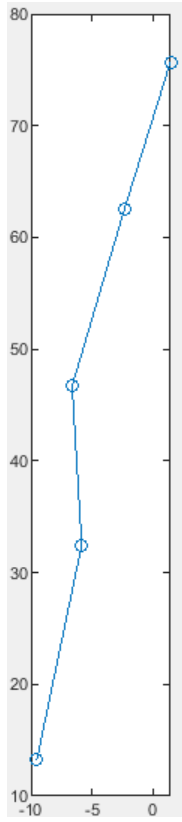

(b) Normal Posture

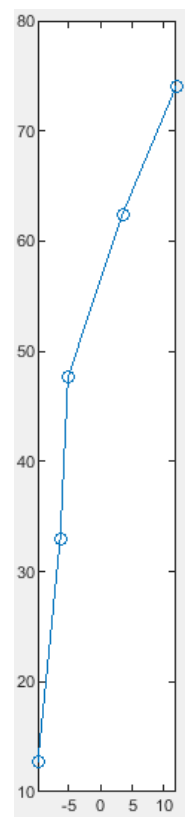

(c) Slouched Posture

Figure 10. Simplified spine model (lumbar thoracic and cervical region) in sagittal plane by MATLAB

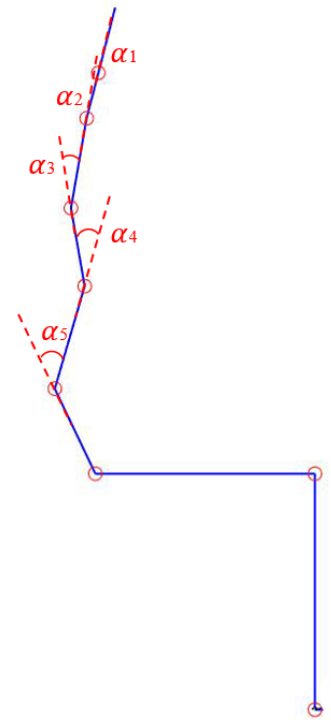

Figure 11. The definition of the turning angles at each joint on the simplified spine model

Table 6. joint turning angles for the modeled three postures based on Figure 11

\begin{tabular}{|c|c|c|c|}
\hline Joint angle & Erect posture & Normal posture & Slouched posture \\
\hline$\alpha_{1}$ & $-41.6^{\circ}$ & $-46.45^{\circ}$ & $-47.6^{\circ}$ \\
\hline$\alpha_{2}$ & $26.1^{\circ}$ & $13.9^{\circ}$ & $5.1^{\circ}$ \\
\hline$\alpha_{3}$ & $-19.7^{\circ}$ & $-18.3^{\circ}$ & $-25.4^{\circ}$ \\
\hline$\alpha_{4}$ & $-4.73^{\circ}$ & $-0.8^{\circ}$ & $-5.8^{\circ}$ \\
\hline$\alpha_{5}$ & $0.1^{\circ}$ & $0.46^{\circ}$ & $0.1^{\circ}$ \\
\hline
\end{tabular}




\subsubsection{Spine force modelling in sagittal plane}

Based on the results obtained from the last section of modelling the spine curvature and segmentation, the force can be analyzed for the spine when sitting in the chair with the back contacting the seat cushion at the backrest. While the back is leaning on the seat for a comfortable sitting, the body would experience the forces that supports the body so that muscles can be relaxed, and comfort can be generated. In sagittal plane, the supporting force is a combination of friction and contact normal force for each spine region. The friction is tangent to the contact plane; for the simplified body segment that is used for the analysis, the friction direction is aligned with the segment itself. Considering the segment in the middle of the spine, there are forces from the previous spine segment, while there are also forces that hold the segment at this position statically. Of course, the segment is also loaded with its own gravitational force at its center of mass, which locates at the middle of the spine. A free body diagram (FBD) that describes the loadings on one segment is as shown in Figure 12.

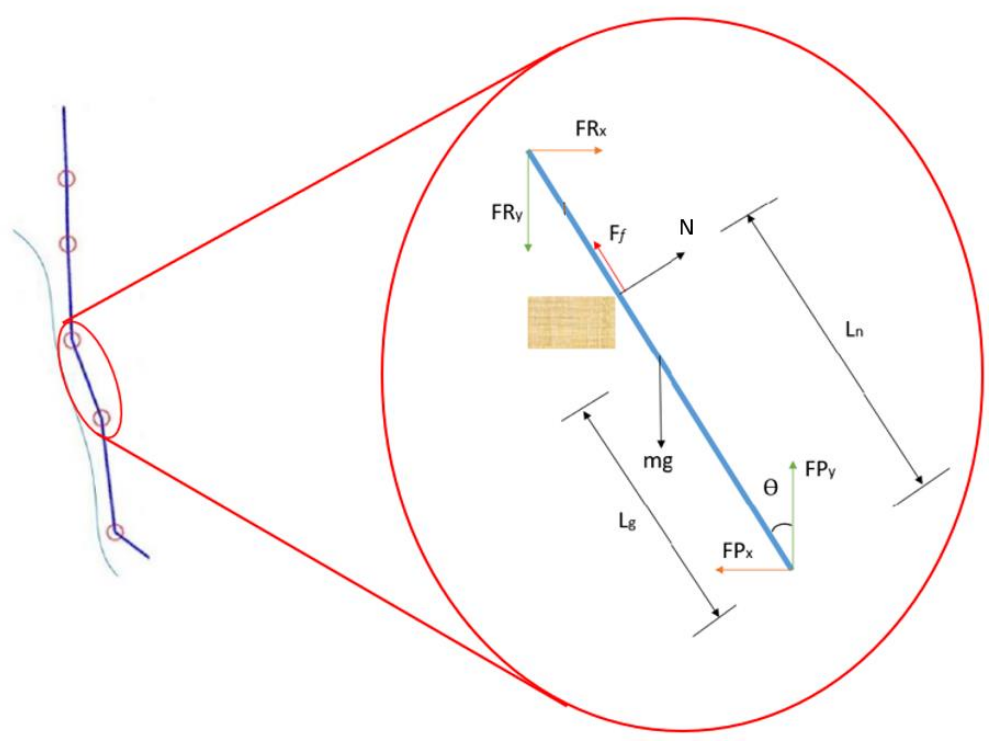

Figure 12. Free body diagram in sagittal plane for one segment that has contact with the seat cushion

The full back (spine) of the human contacts the seat cushion. In this report, however, for the simplicity of the force analysis, point forces were assumed for the loadings on the spine segment. Lg and Ln defines the locations where the gravitational force and contact forces respectively with respect 
to the lower end of the segment. $\mathrm{N}$ and $\mathrm{Ff}$ are the contact normal and friction force acting on the contact point. FR and FP are the loading from the previous top segment and the reaction force from the next bottom segment respectively.

To analyze the FBD as presented in Figure 12, the condition of the spine segment when the passenger is sitting statically and comfortably can be assumed to be static. A static object must have its net force and net moment both balanced as zero.

$$
\sum F=0 ; \quad \sum M=0
$$

For the balanced force and moment, such relationships can be generated (the moment equilibrium is determined referring to the bottom end joint of the segment; $G$ is the gravitational force).

$$
\begin{aligned}
& \sum M=\vec{L} \times \overrightarrow{F R}+\overrightarrow{L_{n}} \times \vec{N}+\overrightarrow{L_{g}} \times \vec{G} \\
& \sum F=\overrightarrow{F R}+\vec{N}+\overrightarrow{F_{f}}+\vec{G}+\overrightarrow{F P}
\end{aligned}
$$

If the friction with the same direction of the link (pointing to the upward direction) can be determined by

$$
\overrightarrow{F_{f}}=\mu|\vec{N}| \frac{\vec{L}}{|\vec{L}|}
$$

The friction coefficient at the contact interface is assumed to be a constant of 0.15 [10]. The contact point on this segment is assumed to locate at the middle point, same as the location of center of gravity. So, the length vector has such relationship

$$
\overrightarrow{L_{n}}=\overrightarrow{L_{g}}=\frac{1}{2} \vec{L}
$$

The gravitational forces values of different segment masses are based on the results gained by Ghezelbash and coworkers [20]. In their research, the segmental weight increase for BMI of 30, 35 and 40 
compared to the segmental body weight of persons with BMI 25 was found. For our participants who has the BMI values that are not exactly those studied by Ghezelbash and coworkers, the linear interpolation method was applied in order to calculate the body segment weight of the person with his/her BMI that falls in between the exact analyzed values $(25,30,35,40)$. Their results data is utilized in this report for the weight estimation for the segmented region of the spine. With the body weight information (as presented in Table 7) derived from the data provided by Ghezelbash, the vector of the gravitational force of the segment $\vec{G}$ can be determined. Note that the weights of the arm are also counted, and they were additional mass of thoracic I region; the values presented in the thoracic I column are the summed weight. As the segmented model has been obtained, all the length vector become known variables for solving equation (7), where $\overrightarrow{F R}$ is derived from the previous segment's force analysis, and the gravitational force of the object segment (direction is vertically downwards). Therefore, the only unknow in equation (7), the normal force $\vec{N}$ can be solved with its known direction that is perpendicular to the segment.

$$
\vec{N} \cdot \vec{L}=0
$$

Table 7. head mass and segmental mass of the spine for cases with BMI of 25, 30, 35, 40

\begin{tabular}{|r|c|c|c|c|c|}
\hline \multirow{2}{*}{ BMI } & \multicolumn{5}{|c|}{ Mass (kg) } \\
\cline { 2 - 6 } & Head & Cervical & Thoracic I & Thoracic II & Lumbar \\
\hline $\mathbf{2 5}$ & 2.27 & 0.93 & 14.4 & 9 & 8.6 \\
\hline $\mathbf{3 0}$ & 2.525 & 1.09 & 17.3 & 11.8 & 11.81 \\
\hline $\mathbf{3 5}$ & 2.882 & 1.27 & 19.86 & 14.79 & 15.53 \\
\hline $\mathbf{4 0}$ & 3.238 & 1.45 & 22.36 & 18.32 & 19.25 \\
\hline
\end{tabular}

For segments that are not contacting with the seat cushion and thus do not have support on the spine segment, the moment generated by the body weight must be balanced by the functioned muscle and ligament. Therefore, no moment equilibrium would be available for the calculation of the unsupported spine segment. The reaction force at the bottom joint $(\overrightarrow{F P})$, which equals in value (but opposite direction) to the 
force that passes to the next lower segment, can be found by summing of the weight of this segment and the net forces passed down from the previous segment.

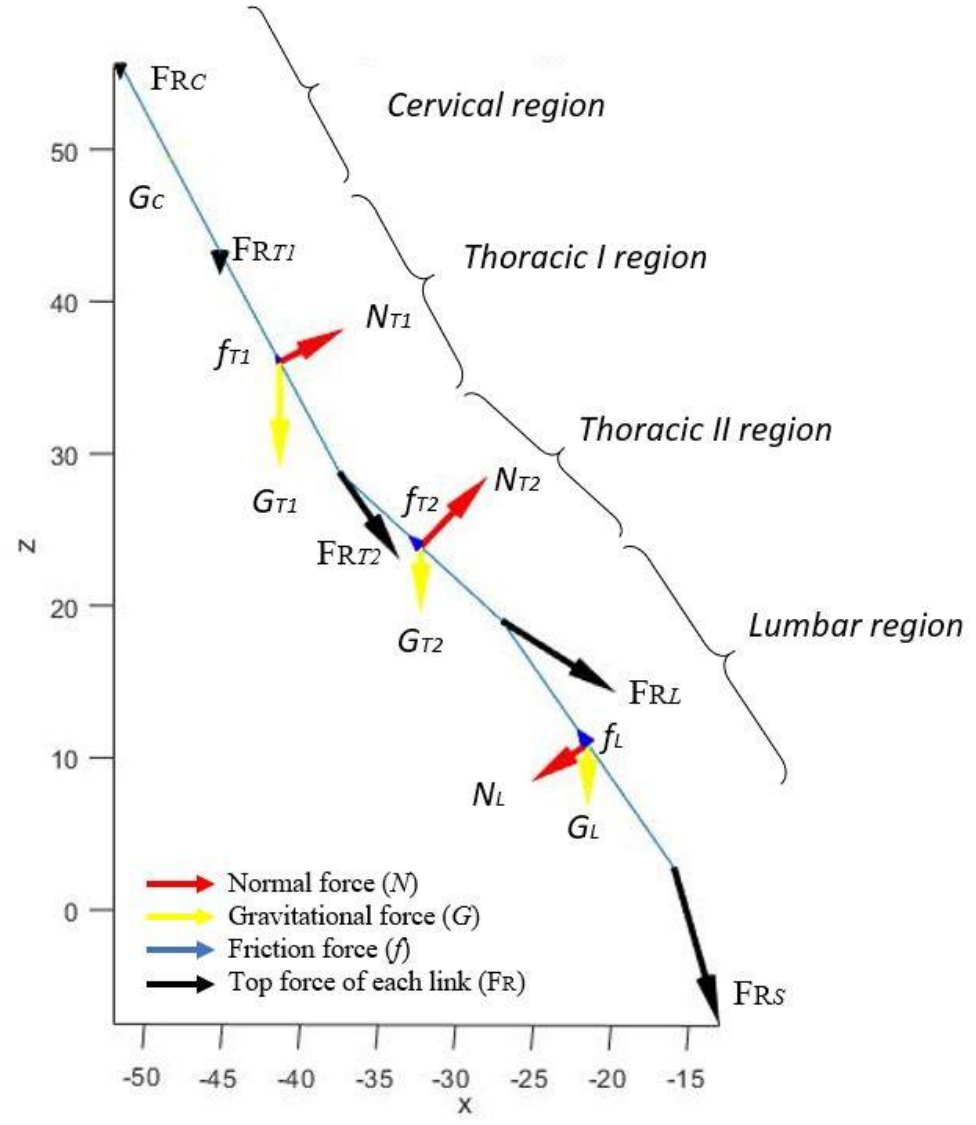

Figure 13. Sample force diagram of the spine (unit: $\mathrm{cm}$ )

For the analysis presented in this report, it is assumed that the seat's backrest provides support mainly to the back regions, which means that the contract between the human body and the seat cushion does not include the head and the neck region (cervical region). Therefore, the described calculation through the equilibrium equations (equation 7 to equation 10) for the spine segment will be applied for thoracic I, thoracic II and lumbar region referring to Figure 9. Based on the modeling and computing method as described for the forces on the spine in sagittal plane, the diagram is as shown in Figure 13, which is a sample diagram representing the forces acting on the spine with the body assumed to have a BMI of 25 at normal posture (referring to Figure 10) and the backrest recline angle is 135 degrees; the backrest recline angle is the angle between the seat pan and the backrest as shown in Figure 14. From Figure 13, the yellow 
arrows represent the gravitational force. Each of the four regions has its weight acting at each link's center point. $G_{c}$ (the weight of cervical region) is not visible because its magnitude is too small comparing with the weight of other segments. The $F_{R}$ force at the joint equal to the summation of the all forces acting on the link above, and it can also be observed that the $F_{R}$ force increases gradually throughout the spine from the top to the bottom due the to cumulative weight of the body from head to lower body. In the scenario as presented in Figure 13, the normal forces supporting the back at thoracic I and II region are pointing to the front side of the body, while the normal force required at the lumbar to balance the spine is pointing to the rear side of the body. Comparatively, the friction forces on the spine is minor, and do not affect the overall spine loading much. Figure 13 is just an example to help illustrate all forces considered in this study. The same calculation is repeated to find how the forces on the spine vary for different cases (varying posture and backrest recline angle). The force diagrams of all studied cases are displayed in the Appendix (Figure 32, Figure 33, Figure 34). The cases and the corresponding results (normal force at the three spinal regions) are presented in the charts below.

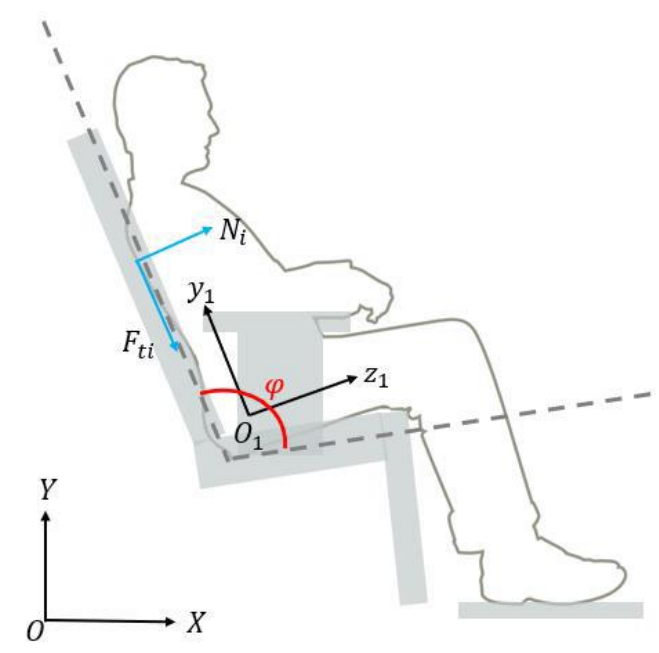

Figure 14. Picture of a sitting human and illustration of backrest recline angle $(\varphi)$ 


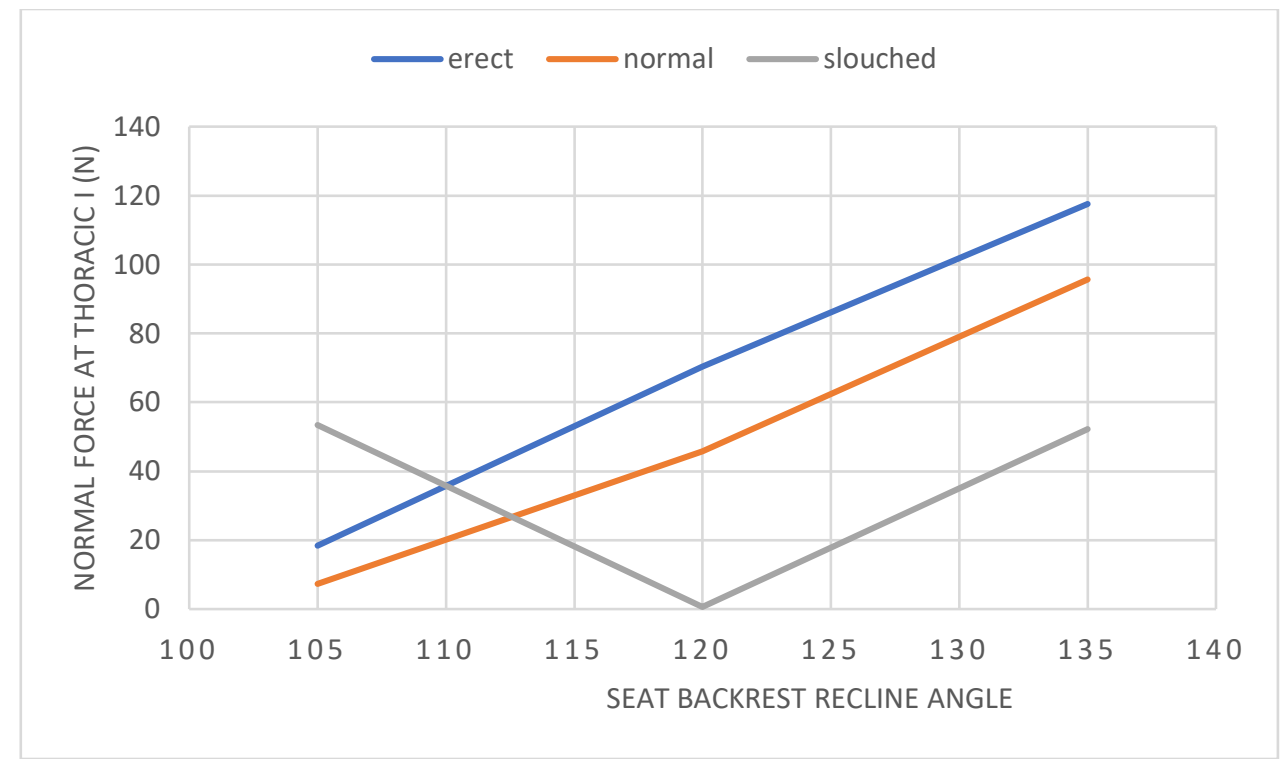

Figure 15. The calculated thoracic I normal force magnitude vs. seat backrest recline angle

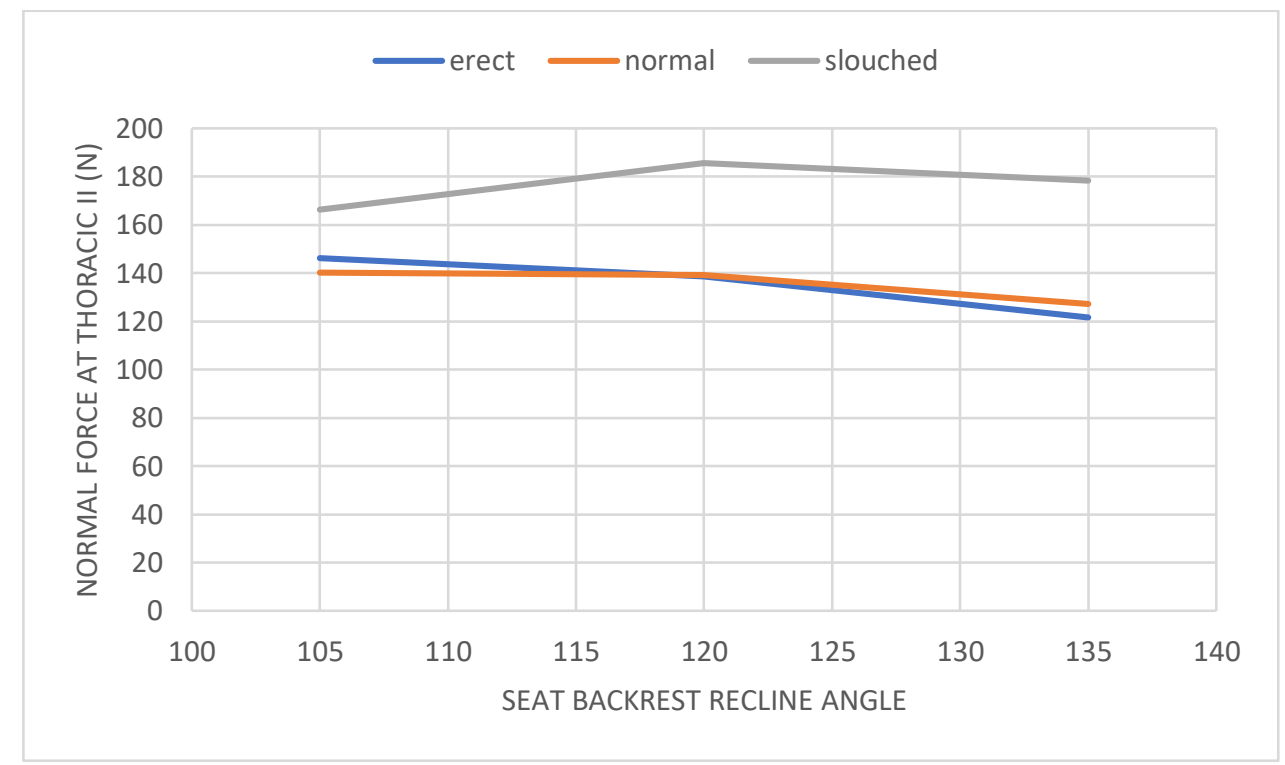

Figure 16. The calculated thoracic II normal force magnitude vs. seat backrest recline angle 


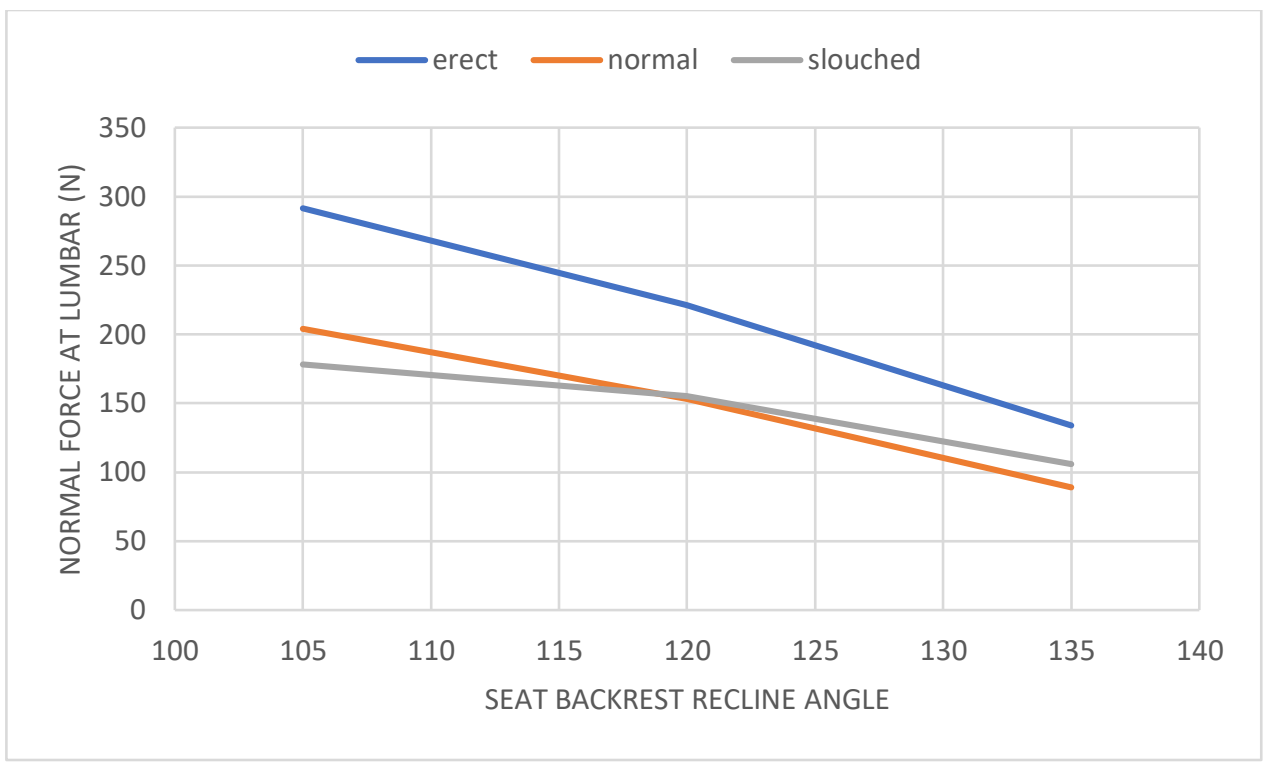

Figure 17. The calculated lumbar normal force magnitude vs. seat backrest recline angle

\subsection{Three-dimensional Modeling}

Based on the modelling of the two-dimensional spinal segments, a three-dimensional model that presents the characteristic of the spine's deformation with more posture cases can be generated. Alike the previous chapter of two-dimensional modelling, the forces on the spine will also be analyzed and modeled in this chapter of three-dimensional modeling.

\subsubsection{Spine Curve Modeling in a Three-dimensional Space}

As presented in section 3.2.2, the spine has already been modeled in the sagittal plane, which is only a two-dimensional model. The three-dimensional space can be created in with two unparallel planes. This section will firstly introduce the modeling of the spine in coronal plane, which is perpendicular to the sagittal plane. The combination of the curve models in the two planes will generate a spine model in a threedimensional space as a result. 
The lateral bending of the spine is achieved by the rotation of each vertebra around the joint on the coronal plane. Figure 18 is a simple serial linkage model that is made of connected length segments.

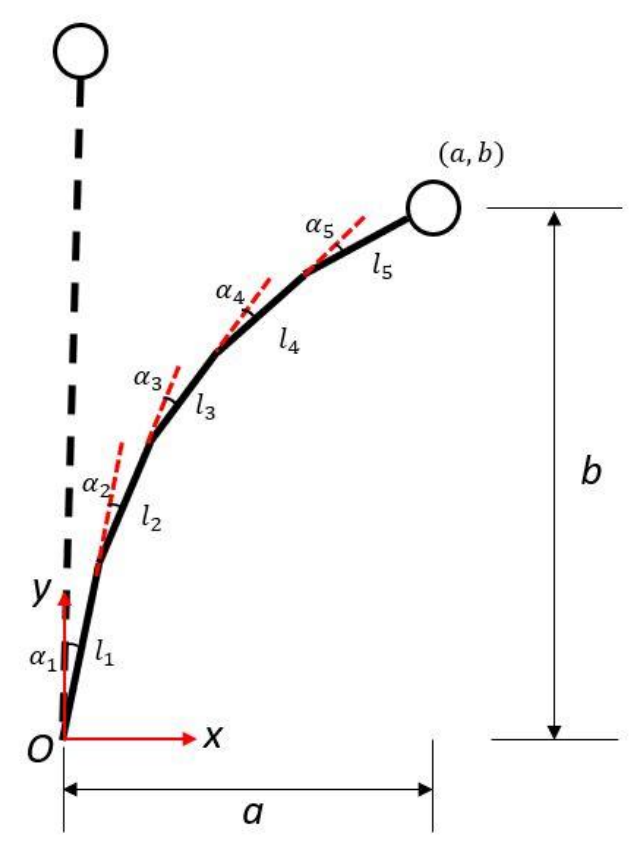

Figure 18. simple serial linkage bending diagram

This simple model can briefly simulate the spine model in terms of concept. The circle at the top represents the interested node whose new location after the bending is to be found; and new location of the node is presented as $(a, b)$ with the origin being set to the root of the linkage (point $O$ ). Such relationships can then be obtained.

$$
\begin{aligned}
& \quad a=l_{1} \sin \left(\alpha_{1}\right)+l_{2} \sin \left(\alpha_{1}+\alpha_{2}\right)+\cdots+l_{5} \sin \left(\alpha_{1}+\alpha_{2}+\alpha_{3}+\alpha_{4}+\alpha_{5}\right)= \\
& \sum_{i=1}^{5}\left(l_{i} \sin \left(\sum_{i=1}^{i} \alpha_{i}\right)\right) \\
& \quad b=l_{1} \cos \left(\alpha_{1}\right)+l_{2} \cos \left(\alpha_{1}+\alpha_{2}\right)+\cdots+l_{5} \cos \left(\alpha_{1}+\alpha_{2}+\alpha_{3}+\alpha_{4}+\alpha_{5}\right)= \\
& \sum_{i=1}^{5}\left(l_{i} \cos \left(\sum_{i=1}^{i} \alpha_{i}\right)\right)
\end{aligned}
$$

For modeling the spine, the length segments presented in Figure 18 are replaced with the lengths of the vertebras units, and the angles are defined by the rotational neutral zone of the vertebra, which has been justified referring to section 3.1.1 about considering the neutral zone of the spine joint motion range). 
Average neutral zone of each vertebra has been collected in Table 4. The length of each vertebra can be estimated with the data provided from Table 9; the difference between the adjacent vertebra centers is treated as the projective length of the vertebra on the coronal plane. For the consistency with the twodimensional segmented spine modeling in section 3.2, the same vertebras must be chosen as the joints that defines the four-region segmented model (cervical, thoracic I, thoracic II and lumbar region). Therefore, the points whose locations are interested are at S1, L1, T6 and T1. The method of finding then points locations are similarly to find the coordinate of $(a, b)$ using equation (12) and (13). The origin is set to the Ischial tuberosities (IT) referring to Figure 9. With all the knows, the lateral bended segmented spine within the neutral range of motion can be determined. In this report, two cases of the lateral bending are focused. One is at the boundary of the neutral zone (referring to the data in the second column of Table 4), which represents the maximum bended condition of the spine without stretching too much and affecting the sitting comfort. The other one is "slight bending", which uses one half of the neutral angles provided in Table 4. The postured spine model in coronal plane are as shown in Figure 19.

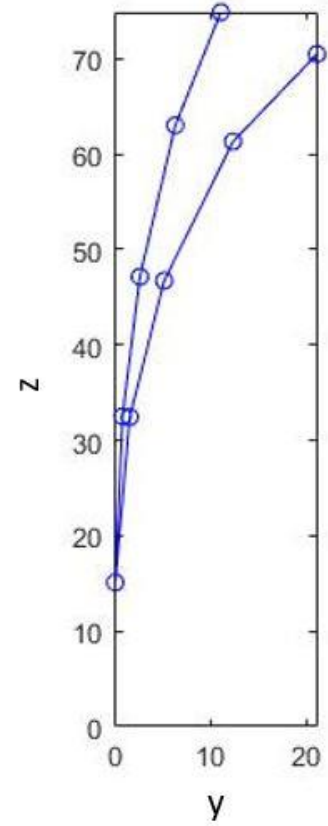

(a)

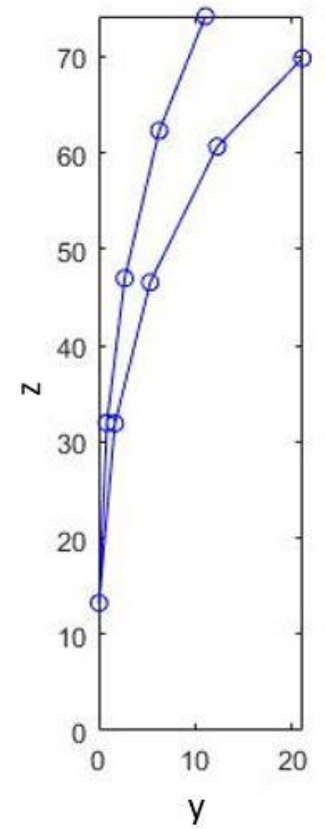

(b)

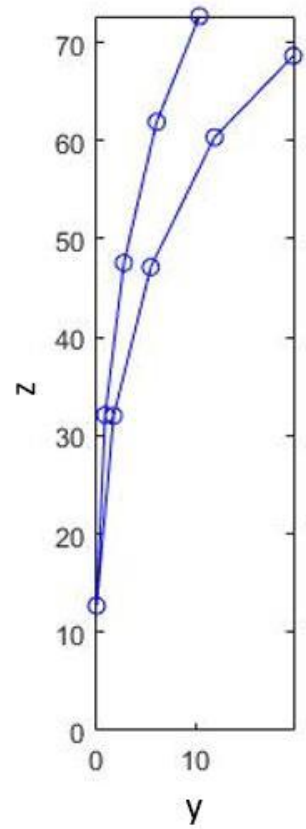

(c)

Figure 19. The segmented spine with two levels of lateral bending (half neutral zone "slight bending" and neutral zone maximum bending) on coronal plane (unit: $\mathrm{cm}$ ) (a) with the erect posture on sagittal plane (b) with the normal posture on sagittal plane (c) with the slouched posture on sagittal plane 
Up to this point, the segmented spine models in sagittal and coronal plane are obtained. The threedimensional modeling can be generated by combining the models built within these two unparallel planes. Figure 20 displays the results of the three-dimensional model of the spine at various postures.

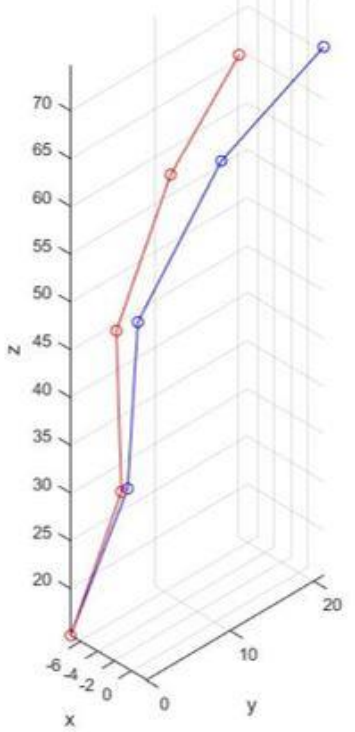

(a)

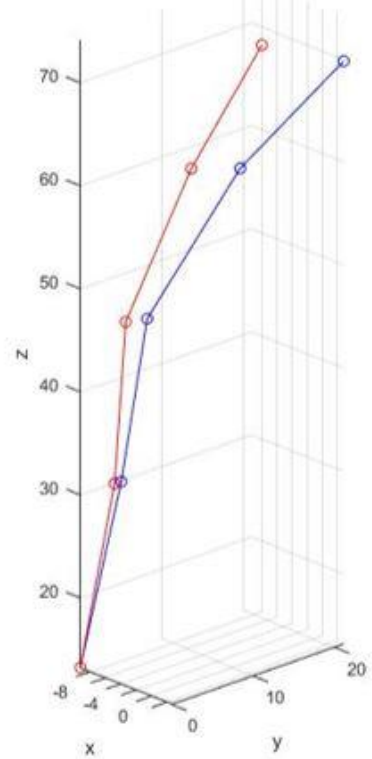

(b)

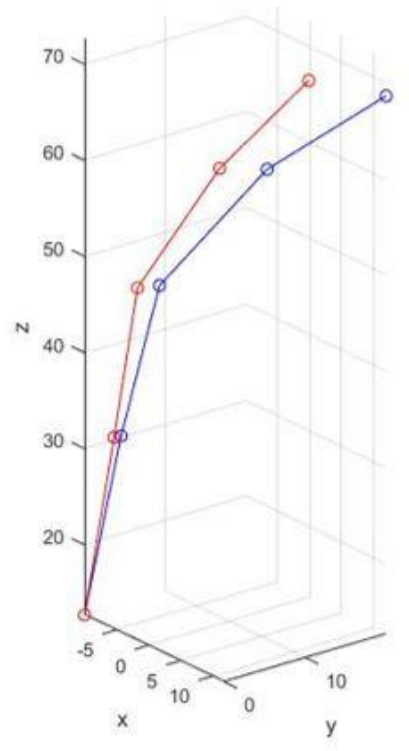

(c)

Figure 20. The three-dimensional segmented spine model with two levels of lateral bending (half neutral zone "slight bending" shown in red and neutral zone maximum bending shown in blue) when the backrest angle is 90 degrees (unit: $\mathrm{cm}$ ) (a) with the erect posture on sagittal plane (b) with the normal posture on sagittal plane (c) with the slouched posture on sagittal plane

\subsubsection{Capturing the Back Shape in Transverse Plane}

The spine shape or spine curve is one important feature of the back, but it cannot completely define the back and be treated as the back model required for the contact pressure distribution as other than the vertebra column, the back surface is formed with the composition of muscle, skin, fat, etc., which could vary much from people to people. Therefore, the case-dependent difference would be hard to modelled generally unlike modeling the spine curve, or further researches on back shape deformation is needed. The back shape of the human body is captured as the shapes that appear in the transverse planes at different 
levels of the body. The level of the cutting plane is based on the segmented spine model. This process can be visualized referring to Figure 21

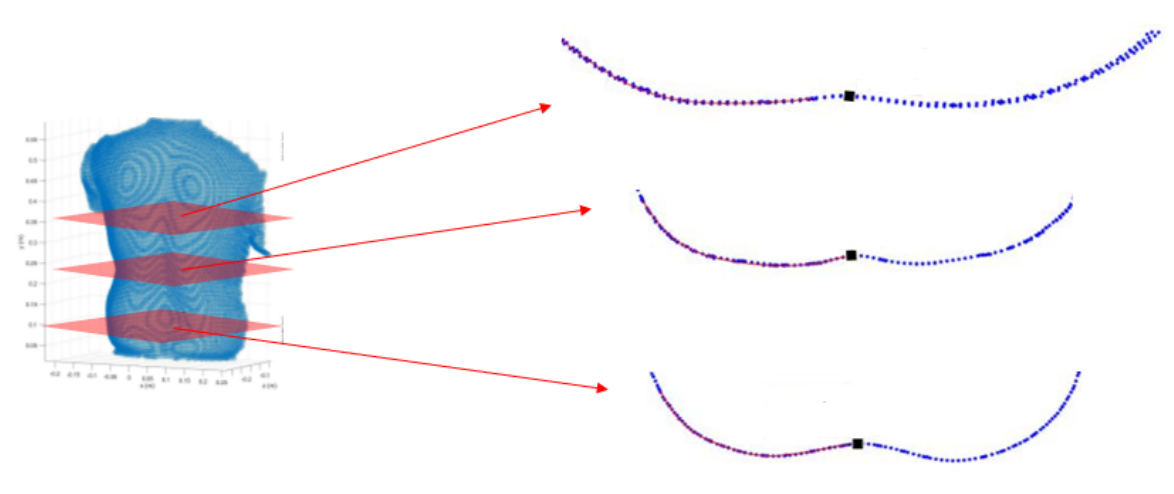

Figure 21. the illustration of obtaining the back shape on transverse plane

In this report, the back shape is captured with actual case study; 5 participants with different body types (with the BMI of 18.8, 20.1, 22.9, 29.1,31.9) were involved. Firstly, their full backs were scanned by portable and wired Sense 3D Scanner developed by 3D System. Then, the scanned documents can be exported with a point cloud format, which allows MATLAB to easily work on. Figure 22 shows the scan image of each subject through MATLAB Figure file by plotting all the point data that was exported from the 3D scanning software. 


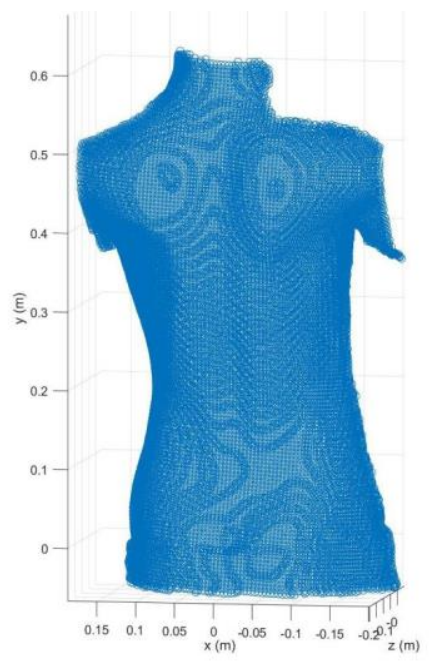

(a)

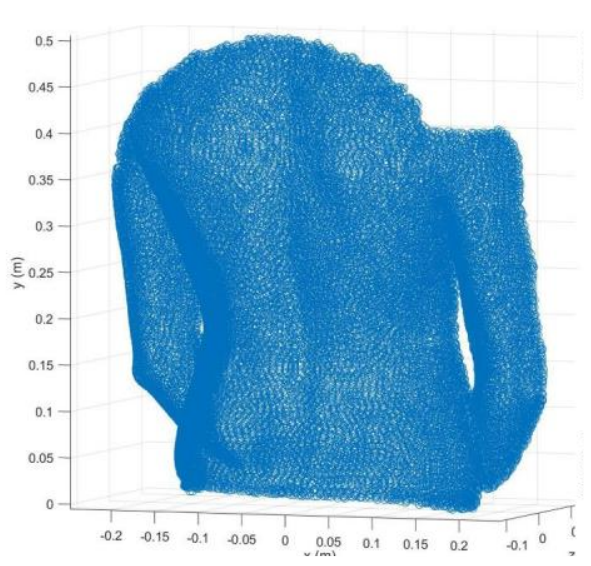

(b)

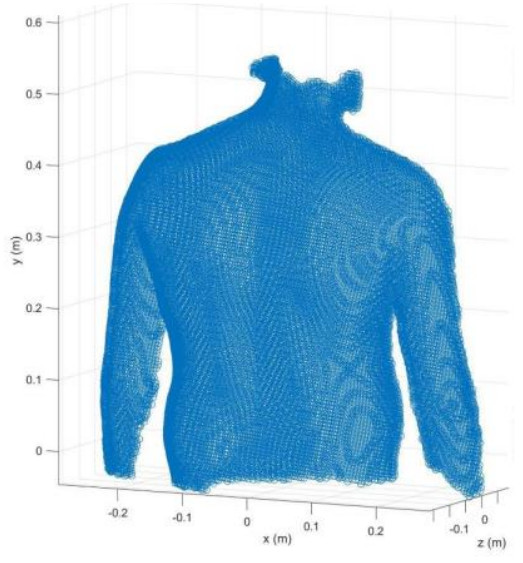

(c)

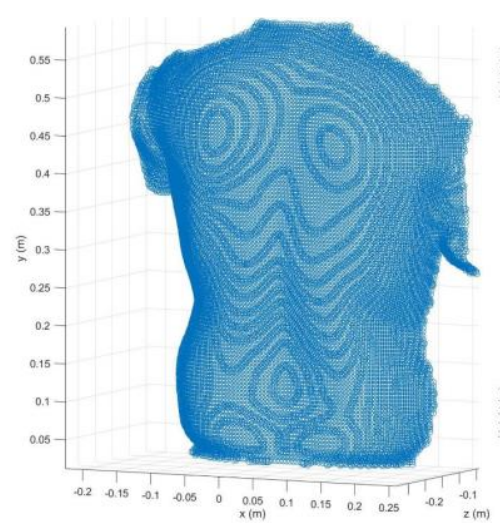

(d)

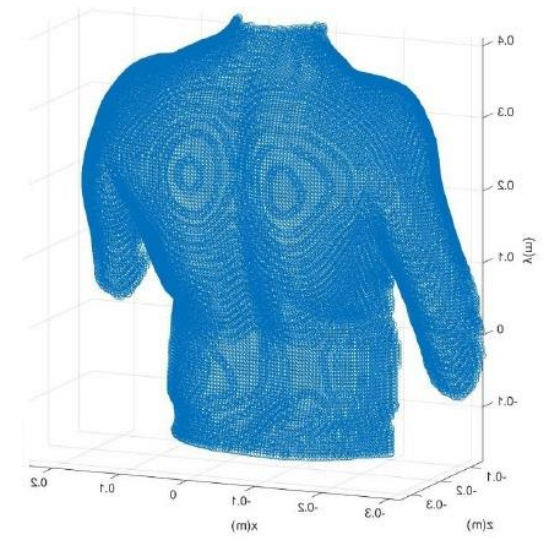

(e)

Figure 22. Collection for the 3D scan figure for each subject. (a) subject 1 with a BMI of 18.8; (b) subject 2 with a BMI of 20.1; (c) subject 3 with a BMI of 22.9; (d) subject 4 with a BMI of 29.1; (e) subject 5 with a BMI of 31.9

For the consistency with the segmented spine model obtained from the previous section, only the shape of back at thoracic I, thoracic II and lumbar is captured instead of the whole scanned model. Therefore, the cutting planes (transverse planes) are set to locate at the middle of the three spine regions. Based on the length ratio of the spine regions, the vertical location of the cutting planes from the bottom joint of the lumbar are listed in Table 8. The general mathematical presentation of a plane in a three-dimensional space can be in such form:

$$
a x+b y+c z+d=0
$$


For the cutting planes (transverse plane that is parallel to $\mathrm{x}-\mathrm{z}$ plane) within the space with the $\mathrm{x}-\mathrm{y}-$ z coordinate of Figure 22, the coefficients in equation (14) are

$$
\left[\begin{array}{l}
a \\
b \\
c \\
d
\end{array}\right]=\left[\begin{array}{c}
0 \\
1 \\
0 \\
-h
\end{array}\right]
$$

Depending on which spine region the transverse plane is determined for, the $\mathrm{h}$ value can be found (presented as $\mathrm{h}_{\mathrm{T} 1}, \mathrm{~h}_{\mathrm{T} 2}$ or $\mathrm{h}_{\mathrm{L}}$ referring to Table 8 for thoracic I, thoracic II and Lumbar region respectively). As a result, all equations for the transverse planes for all subjects can be determined. The scan model is in point cloud format. To find the shape of the body at each of the transverse plane at different spine region, the data points that are close to the planes are kept. The distance between the points to the certain transverse plane is the filtering parameter. If the coordinate of one point of the point cloud is $\left(x_{i}, y_{i}, z_{i}\right)$, the distance between the point and the plane defined by equation (14) is calculated by

$$
d=\frac{\left|a x_{i}+b y_{i}+c z_{i}+d\right|}{\sqrt{a^{2}+b^{2}+c^{2}}}
$$

If the distance value (d) is found to be less than $4 \mathrm{~mm}$, the point is considered close enough to the transverse plane and will be included to get the shape of the back on this transverse plane. As a result, the filtered points form the shape of the back at each transverse plane's level for different spine region, and they are collected and illustrated in Error! Reference source not found. and Figure 31 in the Appendix.

Table 8. Cutting plane location of each subject (unit: $\mathrm{mm}$ )

\begin{tabular}{|c|c|c|c|c|c|}
\hline Spine region & Subject 1 & Subject 2 & Subject 3 & Subject 4 & Subject 5 \\
\hline Thoracic I $\left(\mathrm{h}_{\mathrm{T} 1}\right)$ & 462.0 & 378.0 & 420.0 & 504.0 & 410.0 \\
\hline Thoracic II $\left(\mathrm{h}_{\mathrm{T} 2}\right)$ & 294.0 & 240.3 & 233.0 & 320.4 & 257.0 \\
\hline Lumbar $\left(\mathrm{h}_{\mathrm{L}}\right)$ & 107.0 & 87.8 & 97.5 & 117.0 & 87.5 \\
\hline
\end{tabular}




\subsubsection{Three-dimensional Spine Force Modeling}

Like the completed two-dimensional force modeling in section 3.2.3, the forces acting on the three dimensional spine starts with a single body free body diagram force analysis. The three-dimensional forces on the spine is the combination of the forces in sagittal plane and coronal plane. As the force analysis in sagittal plane has been completed, this section will firstly discuss about the forces on one spine segment in the coronal plane.

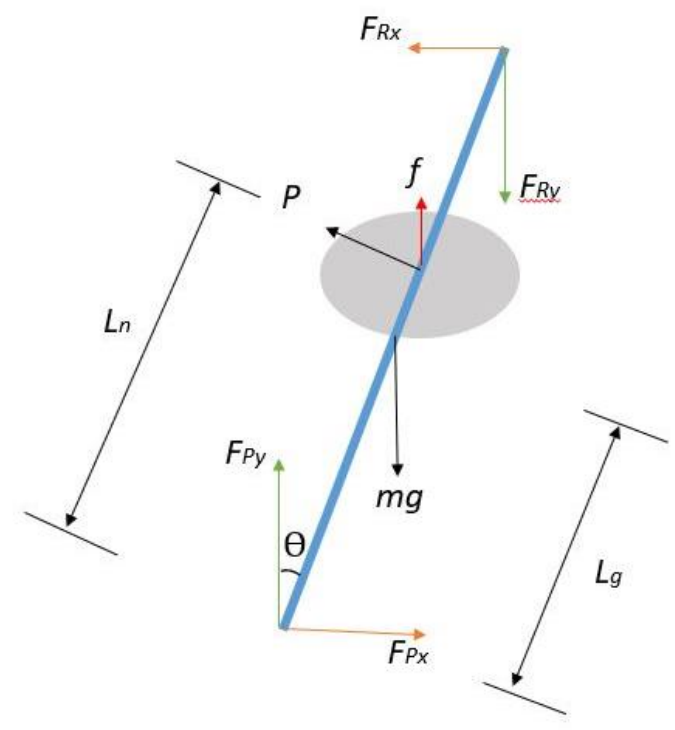

Figure 23. Free body diagram in the coronal plane for one segment that has contact with the seat cushion

As shown in Figure 23, one spinal segment in coronal plane experiences the force at the top end (FR) from the previous segment, and the reaction force at the bottom (FP). Gravitational force, same as FBD in sagittal plane, acts at the mass center of the segment and magnitude is the product of the mass and the gravitational acceleration $(9.81 \mathrm{~m} / \mathrm{s} 2)$. Instead of there being a normal force supporting the spine as in the sagittal plane, in coronal plane a reaction force $(\mathrm{P})$ is assigned at the contact point. The reaction force $\mathrm{P}$ just represents a force that prevents the body segment from rotation around the bottom joint when the spine or body is leaning on the backrest statically; it is why the direction of $\mathrm{P}$ is perpendicular to the segment. Depending on the design of the seat's backrest, the $\mathrm{P}$ can be friction or normal force as well of the backrest pattern has certain curvature, or even both combined. This report doesn't dip into details about the sources 
of the forces, and the reaction force $\mathrm{P}$ is the resultant force due to the contact at the contact point. The grey area in the figure represents the backrest pan surface that contacts with the back of the human. For simplifying the problem, the contact region is assumed to be a point and the reaction forces are seen acting on that point only. the force represented by the red arrow pointing upwards is the friction force $(f)$ that has been calculated in the sagittal plane force analysis (refer to equation (9)). The method to find the reaction force $\mathrm{P}$ and $\mathrm{F}_{\mathrm{P}}$ is identical to that has been presented in section 3.2.3. The moment and force equilibrium relationship are utilized to build the equations to solve the reaction forces. For the moment equation, the reference point is the lower end of the segment as shown in Figure 23.

$$
\begin{gathered}
\sum M=\vec{L} \times \overrightarrow{F R}+\overrightarrow{L_{n}} \times \vec{P}+\overrightarrow{L_{g}} \times \vec{G}+\overrightarrow{L_{n}} \times \vec{f} \\
\sum F=\overrightarrow{F R}+\vec{P}+\overrightarrow{F_{f}}+\vec{G}+\overrightarrow{F P}
\end{gathered}
$$

Same as section 3.2.3, the reaction force $\mathrm{P}$ is assumed to locate at the center of the segment. Therefore, the relationship of the length vectors presented in equation (10) is still valid in the calculation here. Because $\mathrm{P}$ is perpendicular the segment, equation (18) exists. With all the equations, the reaction forces $\mathrm{P}$ and $\mathrm{F}_{\mathrm{R}}$ are the only unknowns and therefore they can be solved.

$$
\vec{P} \cdot \vec{L}=0
$$

At this point, the forces on sagittal and coronal planes are all resolved. The forces on the two perpendicular planes are combined, then will the three-dimensional force model of the spine be generated. With this method, three dimensional force models with the backrest recline angle of 105 degree, 120 degree and 135 degree, and the spine bending at the neutral zone boundary are shown as the result (referring to Figure 24, Figure 25, Figure 26, Figure 27).

Figure 24 is the three-dimensional force diagram; the blue arrow on each segment of the spine is the total force acting on the segment's center point (assumed contact point). The figures after are the magnitude of the total force at that point for different cases. 


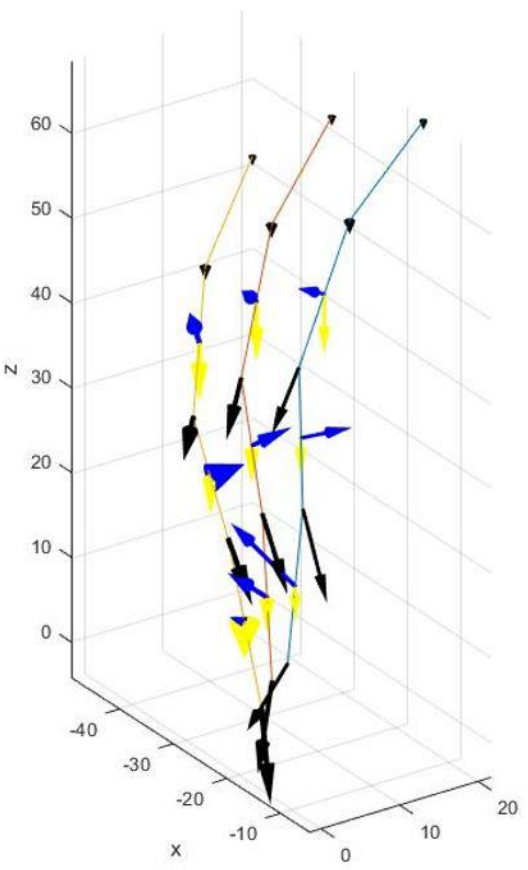

(a)

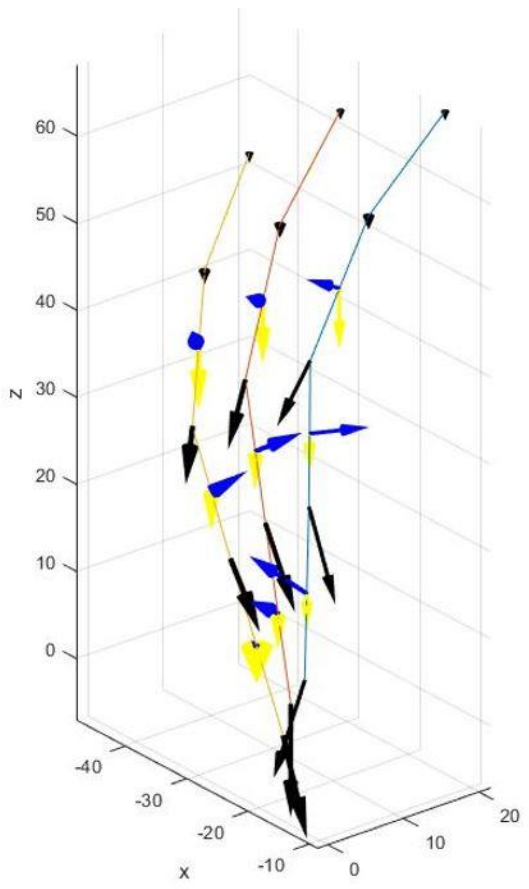

(b)

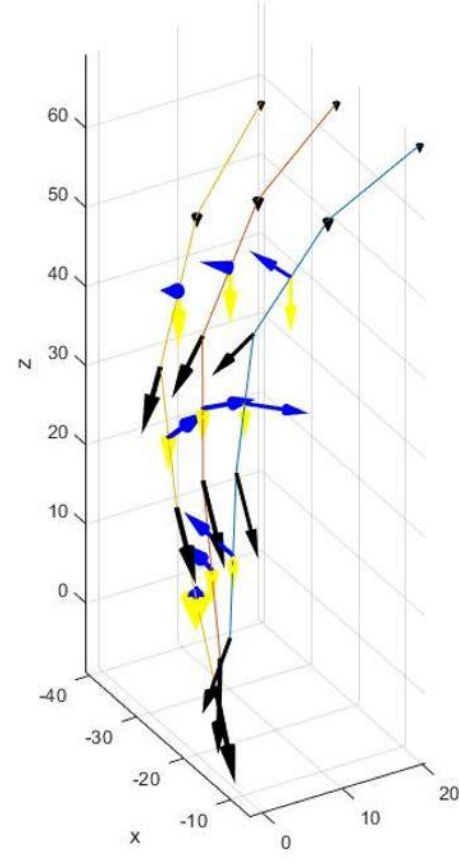

(c)

Figure 24. the force diagram of the spine when the backrest recline angle is 105 degree, 120 degree and 135 degree and the spine bends laterally the most within the neutral zone (axis unit: $\mathrm{cm}$ ) (a) erect posture (b) normal posture (c) slouched posture

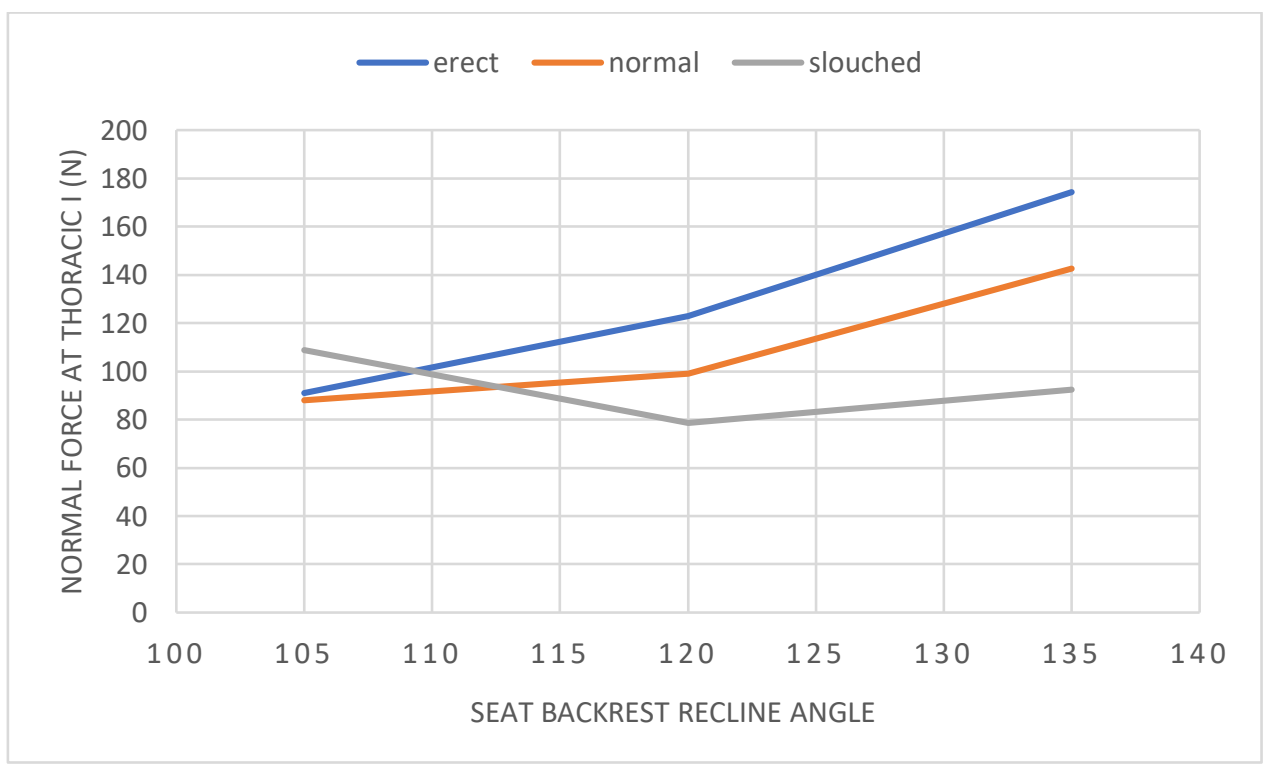

Figure 25. The calculated thoracic I total force magnitude at the contact point vs. seat backrest recline angle 


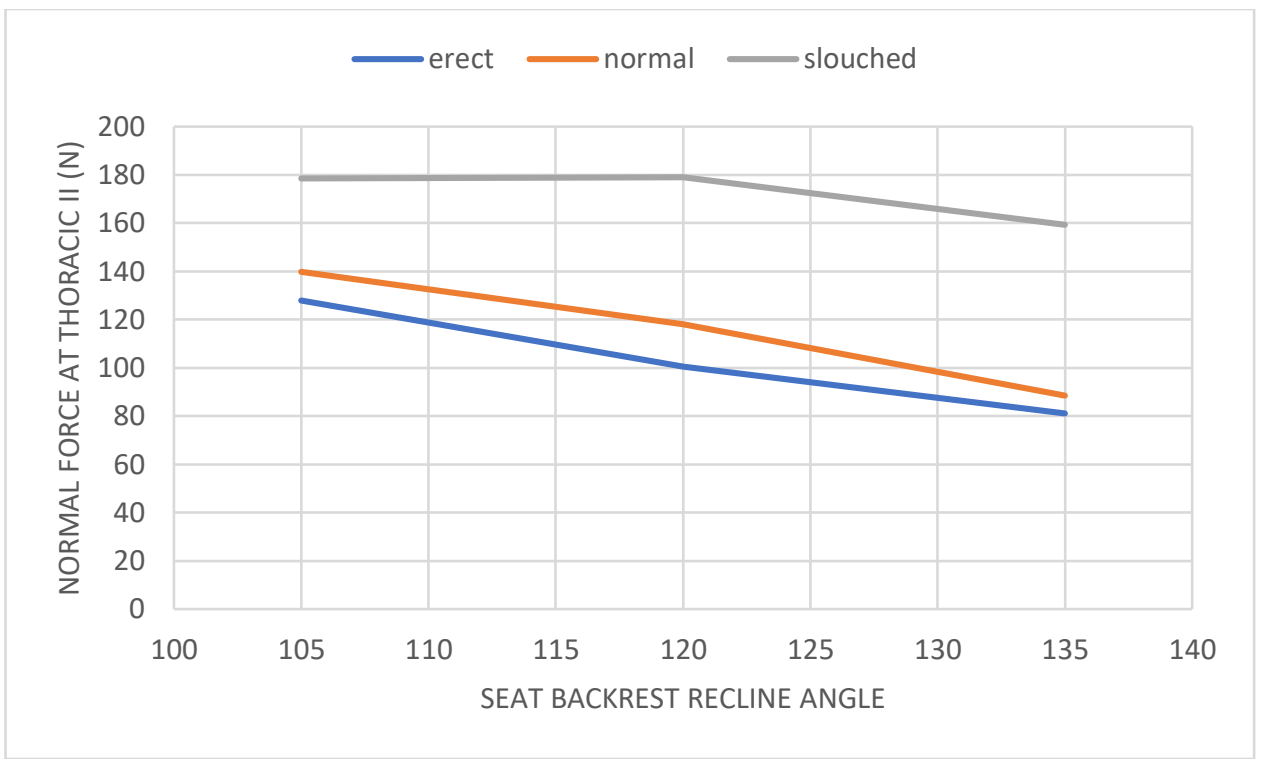

Figure 26. The calculated thoracic II total force magnitude at the contact point vs. seat backrest recline angle

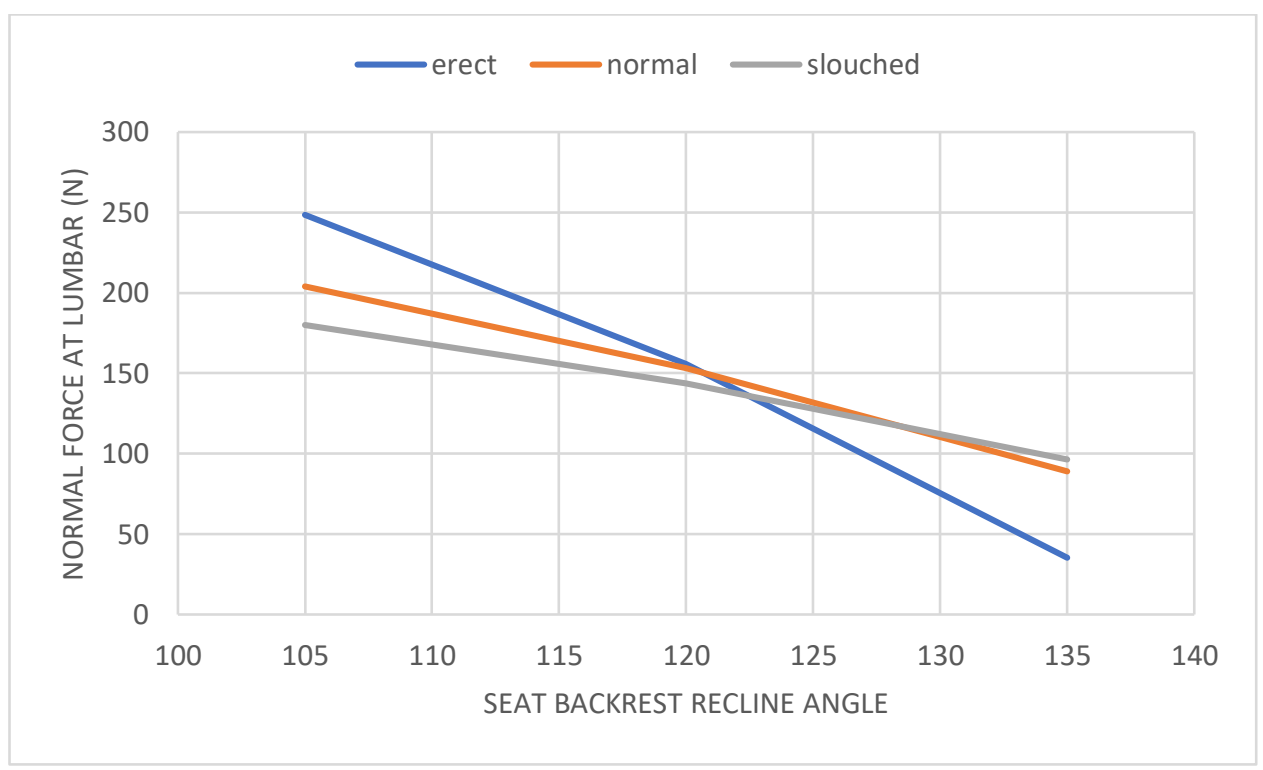

Figure 27. The calculated lumbar total force magnitude at the contact point vs. seat backrest recline angle 


\section{Chapter 4. Conclusion}

The thesis developed a bio model of the human back and spine in all body planes in order to study the pressure condition and comfort due to the backrest of the aircraft seat. Firstly, the concept of comfortability is introduced, as well as the human spine from a perspective of biomechanics. The nature of the spine and the mechanism of the spinal motion were explained as the fundamentals of a proper bio model. Then, two dimensional model was completed in the sagittal plane based on the existing spine data that had been used for spine modelling; three different postures in the same plane were also obtained. The spine model on coronal plane is also built based on the range of motion of each vertebra. Three dimensional spine model is the combination of the models obtained on the sagittal and coronal plane. For the spine model, force analysis is also conducted. The normal and reaction forces acting on each spinal segment are determined, and the diagrams were plotted so that the force scenario can be visualized. In addition, the back shape of the 5 subjects whose body type vary from 'skinny' to 'slight obese' were captured using 3D scanner. They back shape data, and all other modeling work were processed by MATLAB.

A right, and inclusive bio model of the human back and spine will incorporate with the pressure prediction method developed by Liu [10] to obtain a valid and accurate pressure pattern on the seat backrest. This result can then contribute to innovate cushion configuration and new designs of the aircraft seat. 


\section{Reference}

[1] "The Global Commercial Aircraft Cabin Interiors Market." [Online]. Available: https://www.slideshare.net/FrostandSullivan/the-global-commercial-aircraft-cabin-interiorsmarket. [Accessed: 05-Mar-2020].

[2] P. Vink and K. Brauer, Aircraft Interior Comfort and Design. CRC Press, 2016.

[3] M. P. De Looze, L. F. M. Kuijt-Evers, and J. Van Dieën, "Sitting comfort and discomfort and the relationships with objective measures," Ergonomics, vol. 46, no. 10, pp. 985-997, 2003, doi: 10.1080/0014013031000121977.

[4] J. Yongxiang et al., "Sitting posture recognition by body pressure distribution and airbag regulation strategy based on seat comfort evaluation," J. Eng., vol. 2019, no. 23, pp. 8910-8914, 2019, doi: 10.1049/joe.2018.9143.

[5] G. Piretti, P. Srl, and F. S. Mion, "CHAIR WITH SYNCHRONIZED TILTING SEAT AND BACK," vol. 1, no. 12, 2002.

[6] "Lufthansa's new travel class is certainly comfortable, but not cheap-INSIDE Korea JoongAng Daily." [Online]. Available:

http://koreajoongangdaily.joins.com/news/article/article.aspx?aid=3002109. [Accessed: 19-Apr2020].

[7] M. Kolich and S. M. Taboun, "Ergonomics modelling and evaluation of automobile seat comfort," Ergonomics, vol. 47, no. 8, pp. 841-863, 2004, doi: 10.1080/0014013042000193273.

[8] A. Vincent, V. D. Bhise, and P. Mallick, "Seat comfort as a function of occupant characteristics and pressure measurements at the occupant-seat interface," SAE Tech. Pap., 2012, doi: 10.4271/2012-01-0071.

[9] M. Grujicic, B. Pandurangan, G. Arakere, W. C. Bell, T. He, and X. Xie, "Seat-cushion and softtissue material modeling and a finite element investigation of the seating comfort for passengervehicle occupants," Mater. Des., vol. 30, no. 10, pp. 4273-4285, 2009, doi:

10.1016/j.matdes.2009.04.028.

[10] Y. S. Liu, J. Ji, X. Zhong, and J. Xi, "Analysis and Modeling of Human - Seat Interaction Using Bio and Contact Mechanics," 2019.

[11] "Anatomical Terminology | SEER Training." [Online]. Available: https://training.seer.cancer.gov/anatomy/body/terminology.html. [Accessed: 31-Mar-2020].

[12] "Act like a king, sitting on a throne | Physioblogs," Physioblogs, 01-Nov-2015. [Online]. Available: https://physioblogs.wordpress.com/2015/11/01/act-like-a-king-sitting-on-a-throne/. [Accessed: 01-Apr-2020].

[13] "About Adult BMI | Healthy Weight | CDC." [Online]. Available: https://www.cdc.gov/healthyweight/assessing/bmi/adult_bmi/index.html. [Accessed: 01-Apr2020].

[14] Ronan O'Rahilly, Fabiola Müller, Stanley Carpenter, and Rand Swenson, Basic Human Anatomy A Regional Study of Human Structure, Online version. 2004. 
[15] Richard F. LeBlond, Donald D. Brown, Manish Suneja, and Joseph F. Szot, The Spine, Pelvis, and Extremities / DeGowin's Diagnostic Examination, 10th Editi. 2015.

[16] M. M. Panjabi and A. A. White, Basic Biomechanics of the Spine, vol. 7, no. 1. Oxford Academic, 1980.

[17] G. Plaugher and M. A. Lopes, "Clinical Anatomy and Biomechanics of the Spine," Med. Sci. Sport. Exerc., vol. 41, no. January 1993, p. 30, 2009, doi: 10.1249/01.mss.0000352877.48560.b6.

[18] P. A. Oakley, D. D. Harrison, D. E. Harrison, and J. W. Haas, "Evidence-based protocol for structural rehabilitation of the spine and posture: review of clinical biomechanics of posture (CBP) publications.," J. Can. Chiropr. Assoc., vol. 49, no. 4, pp. 270-96, 2005.

[19] S. Kitazaki and M. J. Griffin, "A modal analysis of whole-body vertical vibration, using a finite element model of the human body," J. Sound Vib., vol. 200, no. 1, pp. 83-103, 1997, doi: 10.1006/jsvi.1996.0674.

[20] F. Ghezelbash, A. Shirazi-Adl, N. Arjmand, Z. El-Ouaaid, A. Plamondon, and J. R. Meakin, "Effects of sex, age, body height and body weight on spinal loads: Sensitivity analyses in a subject-specific trunk musculoskeletal model," J. Biomech., vol. 49, no. 14, pp. 3492-3501, 2016, doi: 10.1016/j.jbiomech.2016.09.026.

[21] "Spinal Anatomy New York City | Cervical Spine NYC | Thoracic Spine NY." [Online]. Available: https://www.orthomanhattan.com/conditions-treated-new-york-city/spinal-anatomy/.

[Accessed: 03-Apr-2020]. 
Table 9. Node locations for the head mass, spinal beams and pelvic mass in the three postures

\begin{tabular}{|c|c|c|c|c|c|c|}
\hline \multirow[b]{2}{*}{ Level } & \multicolumn{2}{|c|}{$\begin{array}{l}\text { Erect posture } \\
\left(m \times 10^{-2}\right)\end{array}$} & \multicolumn{2}{|c|}{$\begin{array}{l}\text { Normal posture } \\
\left(m \times 10^{-2}\right)\end{array}$} & \multicolumn{2}{|c|}{$\begin{array}{l}\text { Slouched posture } \\
\left(m \times 10^{-2}\right)\end{array}$} \\
\hline & $x+$ & $z$ & $x$ & $z$ & $x$ & $z$ \\
\hline Head & $2 \cdot 983$ & $80-863$ & $2 \cdot 570$ & $80 \cdot 250$ & 14.856 & $78-157$ \\
\hline $\mathrm{Cl}$ & 1.854 & $76 \cdot 384$ & 1.411 & $75 \cdot 710$ & 11.887 & $74-048$ \\
\hline $\mathrm{C} 2$ & 1.629 & $75-493$ & $1 \cdot 180$ & 74.807 & $11 \cdot 296$ & $73-230$ \\
\hline $\mathrm{C} 3$ & 1.252 & $73-996$ & 0.792 & 73.289 & $10 \cdot 303$ & $71-856$ \\
\hline $\mathrm{C} 4$ & $0-877$ & $72-507$ & 0.407 & $71 \cdot 780$ & $9 \cdot 316$ & $70-490$ \\
\hline C5 & 0.497 & $71-002$ & 0.017 & $70 \cdot 254$ & 8.318 & $69-109$ \\
\hline C6 & 0.088 & $69-379$ & -0.403 & $68 \cdot 609$ & $7 \cdot 242$ & $67-620$ \\
\hline $\mathrm{C} 7$ & -0.303 & $67-827$ & -0.805 & $67 \cdot 036$ & $6 \cdot 214$ & $66-196$ \\
\hline Tl & -0.694 & 66.275 & -1.207 & 65.463 & $5 \cdot 185$ & 64.771 \\
\hline $\mathrm{T} 2$ & -1.421 & $63-572$ & $-2 \cdot 176$ & $62 \cdot 861$ & $3 \cdot 521$ & $62-435$ \\
\hline $\mathrm{T} 3$ & $-2 \cdot 192$ & $60-908$ & $-3 \cdot 179$ & $60 \cdot 297$ & 1.825 & $60-132$ \\
\hline $\mathrm{T} 4$ & -2.952 & $58-195$ & $-4 \cdot 179$ & $57 \cdot 686$ & 0.124 & $57-786$ \\
\hline T5 & $-3 \cdot 608$ & $55-474$ & -5.087 & $55 \cdot 067$ & -1.472 & $55-434$ \\
\hline T6 & -4.094 & $52-625$ & -5.856 & $52 \cdot 325$ & $-2 \cdot 930$ & $52-971$ \\
\hline $\mathrm{T} 7$ & -4.296 & $50-032$ & $-6 \cdot 346$ & $49 \cdot 797$ & $-4 \cdot 135$ & $50-415$ \\
\hline T8 & -4.276 & $47-283$ & $-6 \cdot 648$ & $47 \cdot 117$ & -5.085 & $47-705$ \\
\hline T9 & -4.036 & 44.411 & -6.762 & $44 \cdot 318$ & -5.769 & $44-873$ \\
\hline T10 & $-3 \cdot 591$ & $41-392$ & -6.073 & $41 \cdot 375$ & $-6 \cdot 202$ & $41-897$ \\
\hline T11 & -2.969 & $38-250$ & $-6 \cdot 495$ & $38 \cdot 313$ & $-6 \cdot 407$ & $38-800$ \\
\hline $\mathrm{T} 12$ & $-2 \cdot 364$ & $35-528$ & $-6 \cdot 205$ & $35 \cdot 307$ & $-6 \cdot 436$ & $35-622$ \\
\hline $\mathrm{Ll}$ & -1.792 & $32-519$ & -5.911 & 31.985 & $-6 \cdot 391$ & $32-108$ \\
\hline L2 2 & -1.529 & $29-239$ & -5.795 & $28 \cdot 365$ & -6.437 & $28-280$ \\
\hline L3 & -1.961 & $25 \cdot 748$ & $-6 \cdot 124$ & $24 \cdot 510$ & -6.829 & $24 \cdot 203$ \\
\hline L4 & $-3 \cdot 472$ & $21-933$ & $-7 \cdot 109$ & $20 \cdot 467$ & $-7 \cdot 742$ & $20-077$ \\
\hline L5 & $-5 \cdot 522$ & $18-202$ & $-8 \cdot 420$ & 16.513 & -8.915 & $16-042$ \\
\hline S1 & -7.188 & $15-113$ & $-9 \cdot 488$ & 13.238 & -9.871 & $12-700$ \\
\hline I.T.t & $0-0$ & $0-0$ & $0 \cdot 0$ & $0-0$ & $0 \cdot 0$ & $0-0$ \\
\hline Pelviş & -0.782 & $10-609$ & $-2 \cdot 436$ & $10 \cdot 355$ & $-2 \cdot 794$ & $10-264$ \\
\hline Pelvic angle & \multicolumn{2}{|c|}{$-9-02$ (degrees) } & \multicolumn{2}{|c|}{$0-0$ (degrees) } & \multicolumn{2}{|c|}{1.99 (degrees) } \\
\hline
\end{tabular}

\$ The co-ordinate system has the $x$-axis for the fore-and-aft direction and the $z$-axis for the vertical direction with the origin at the ischial tuberosities.

† Ischial tuberosities.

$\$$ Location of the pelvic mass was adjusted, comparing the vibration mode shapes.

I Initial pelvic angle around the pivot at the ischial tuberosities, with respect to the angle in the normal posture. 


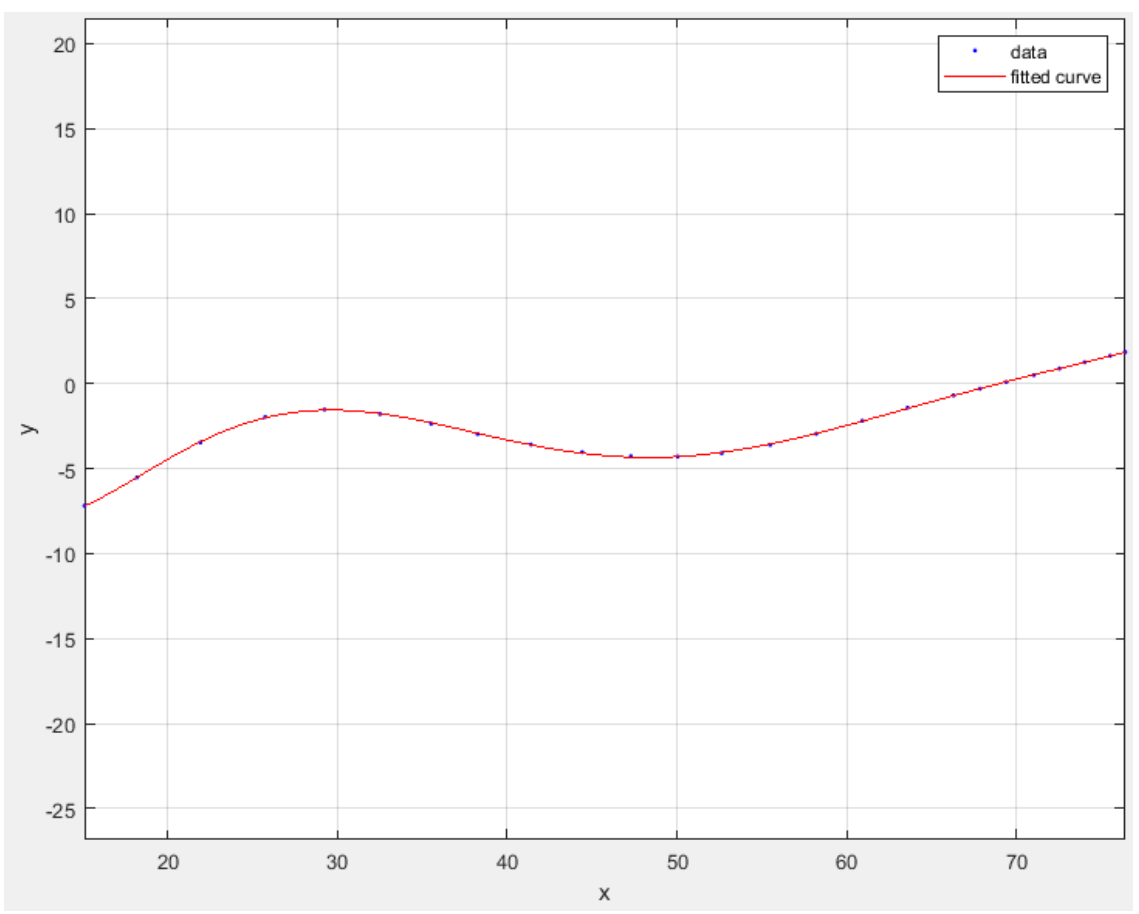

Figure 28. Polynomial fitting curve for a spine with erect posture

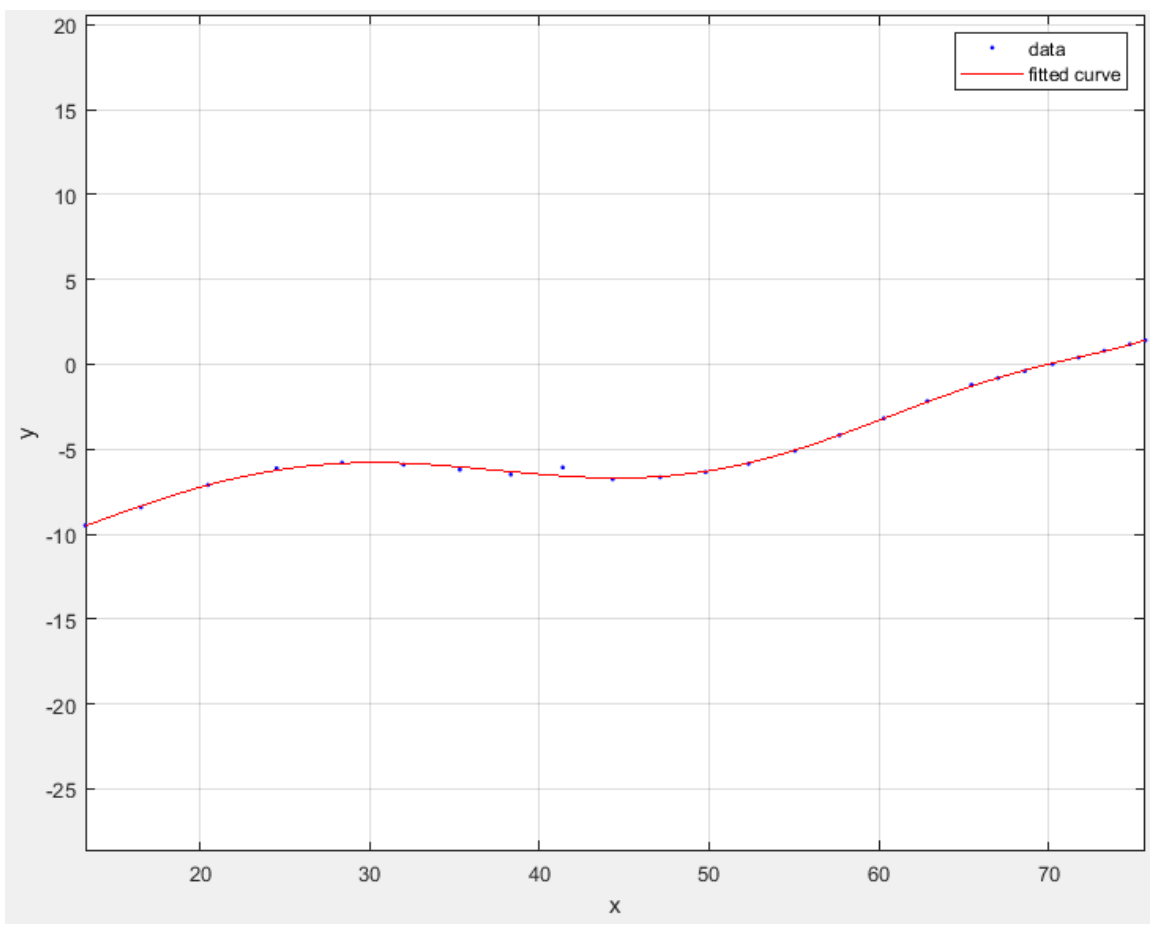

Figure 29. Polynomial fitting curve for a spine with normal posture 


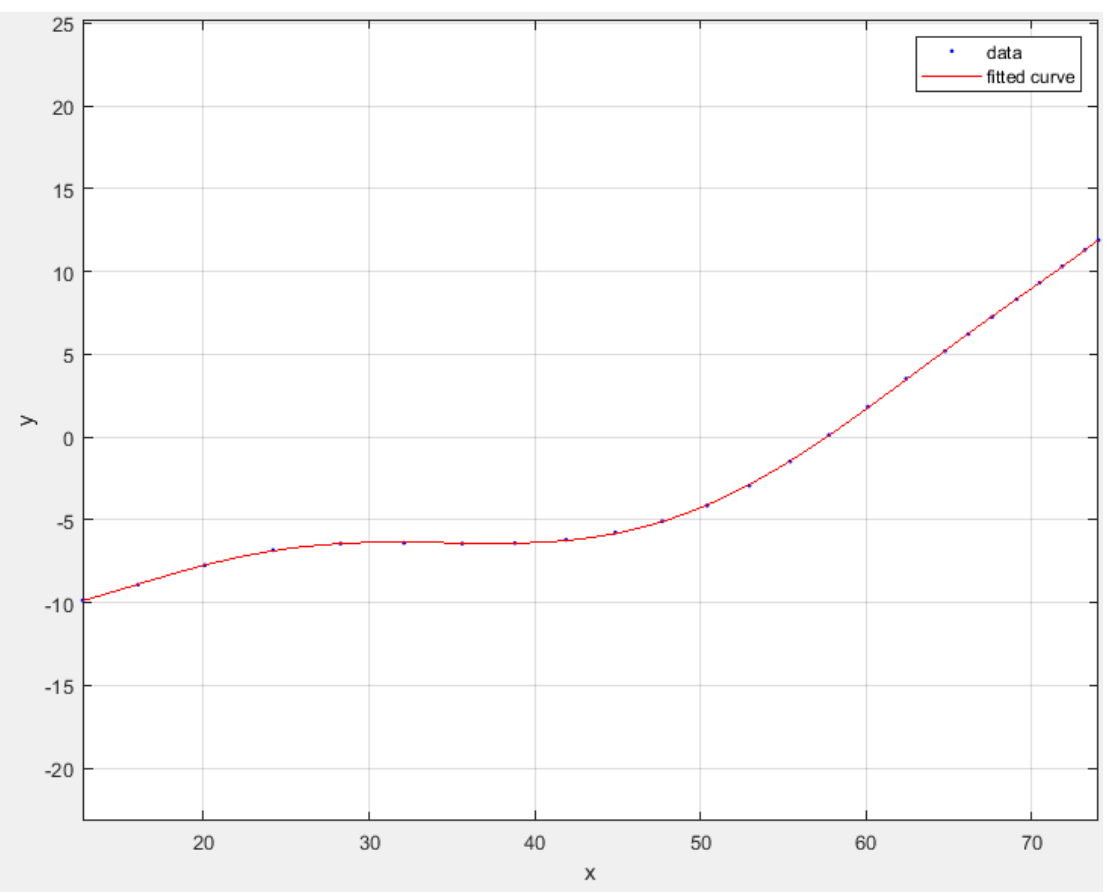

Figure 30. Polynomial fitting curve for a spine with slouched posture 


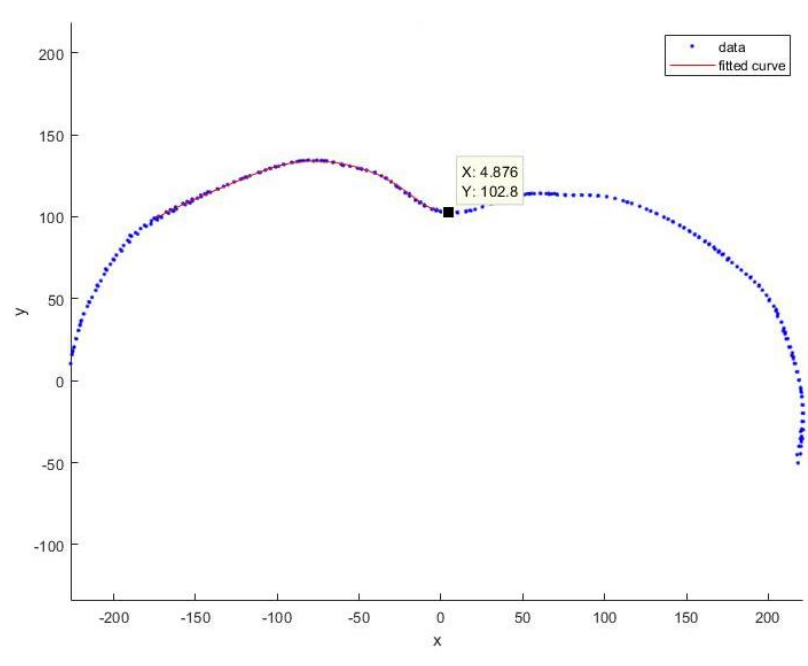

(a)

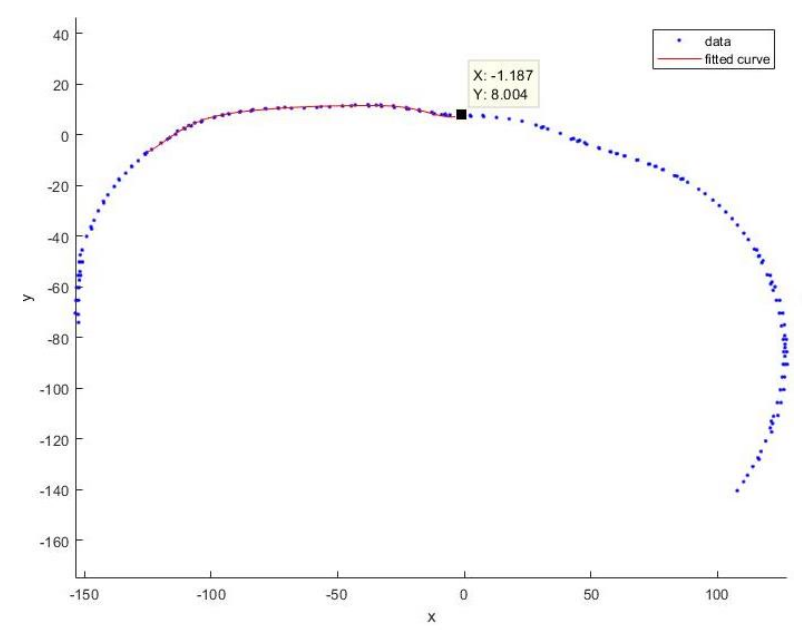

(c)

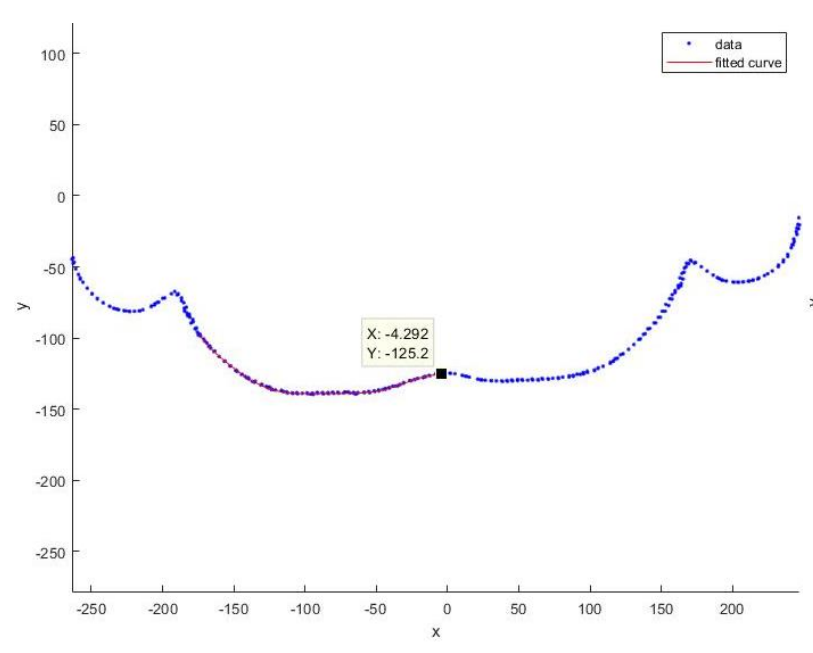

(e)

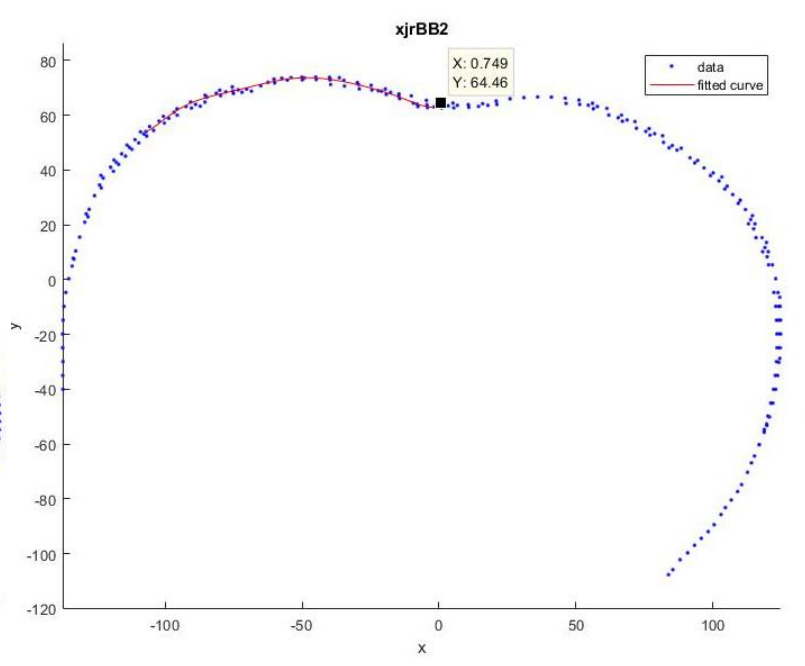

(b)

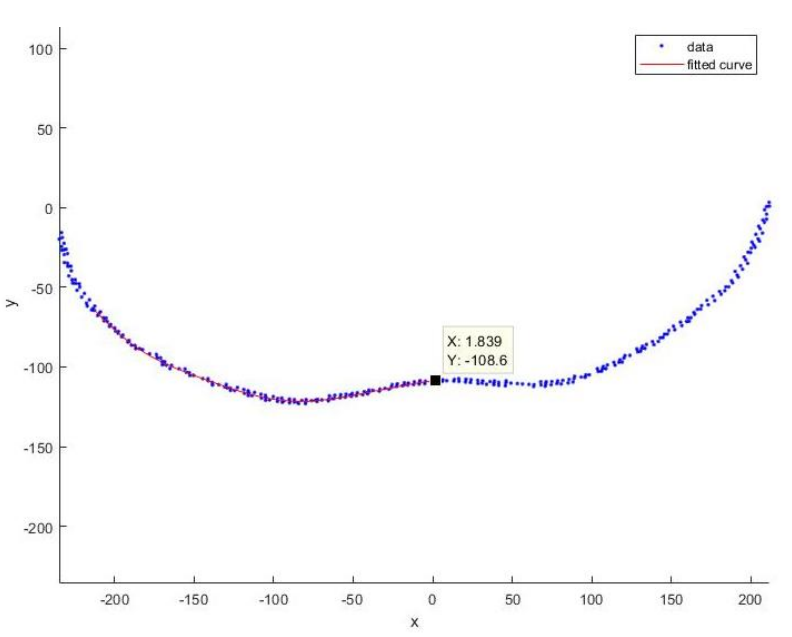

(d)

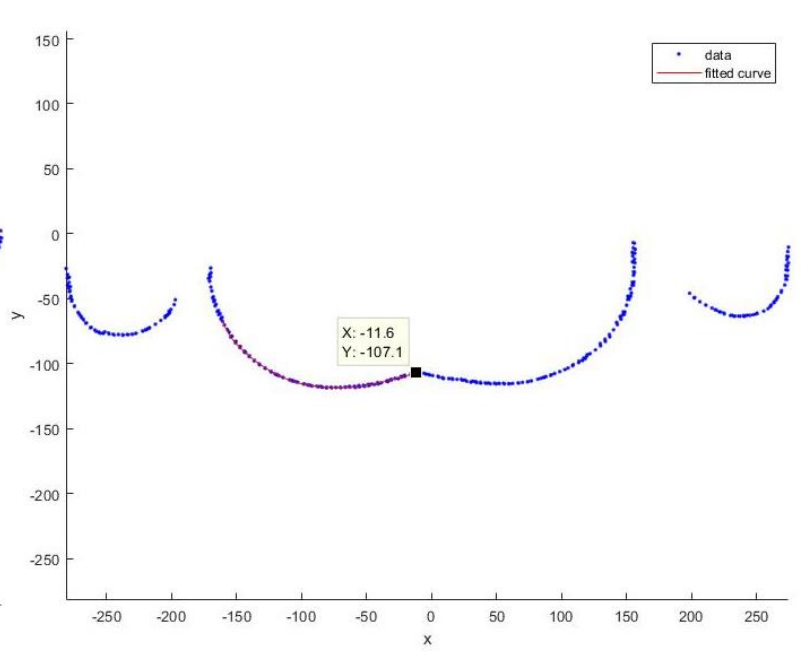

(f) 


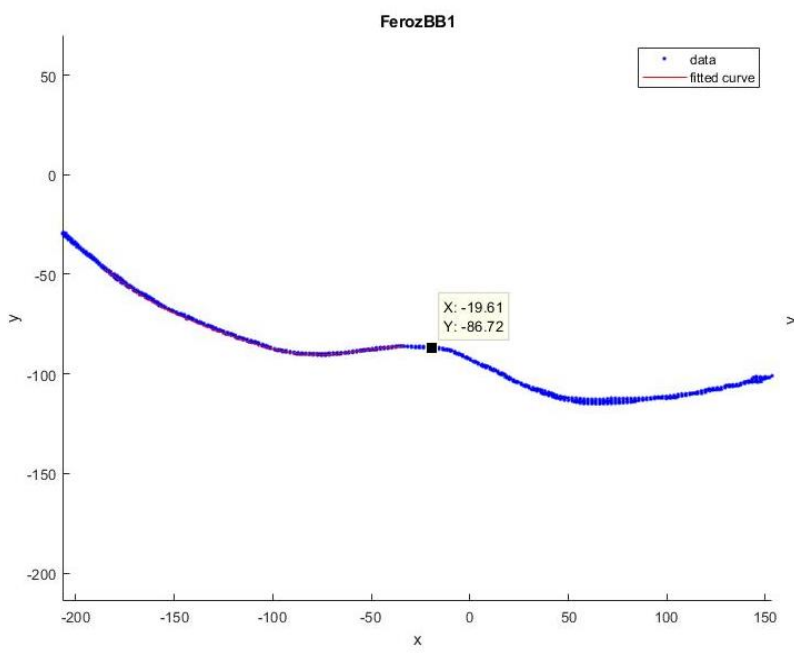

(g)

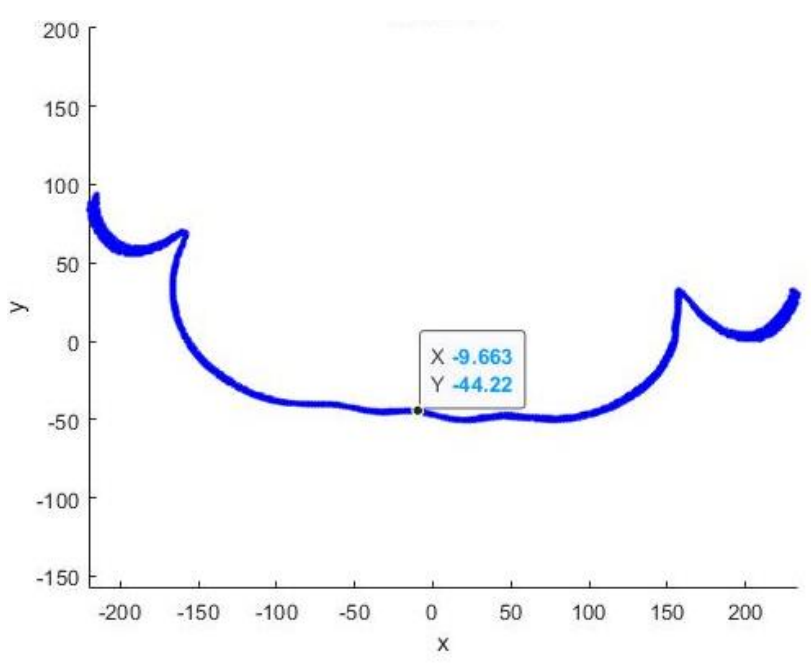

(i)

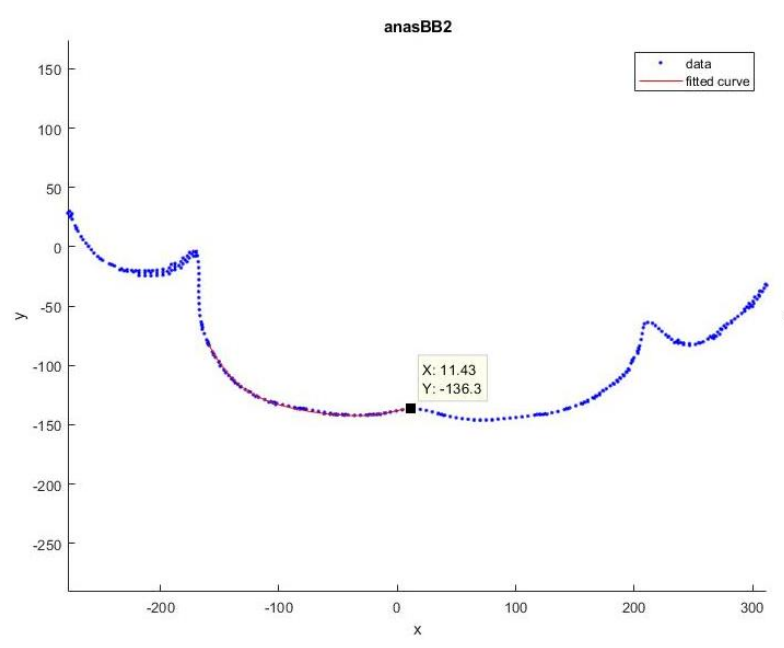

(k)

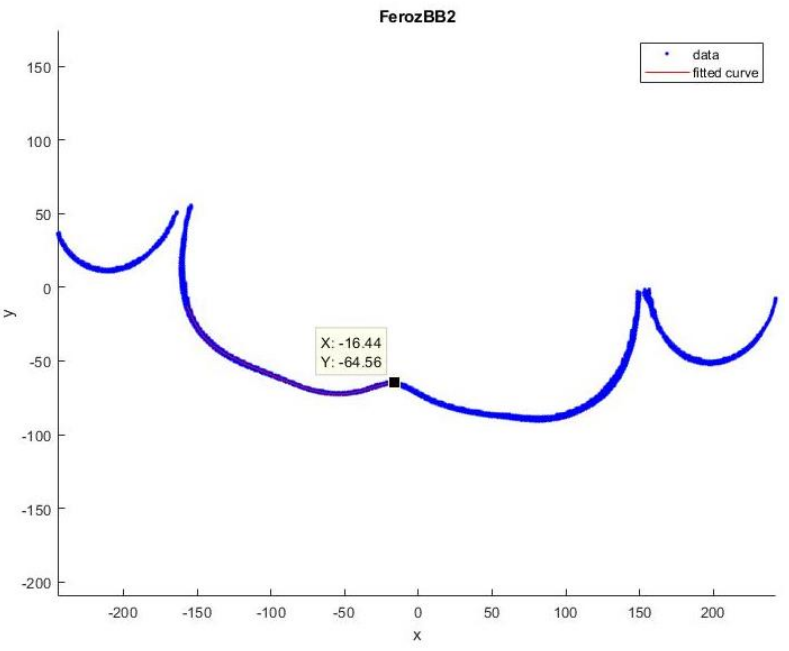

(h)

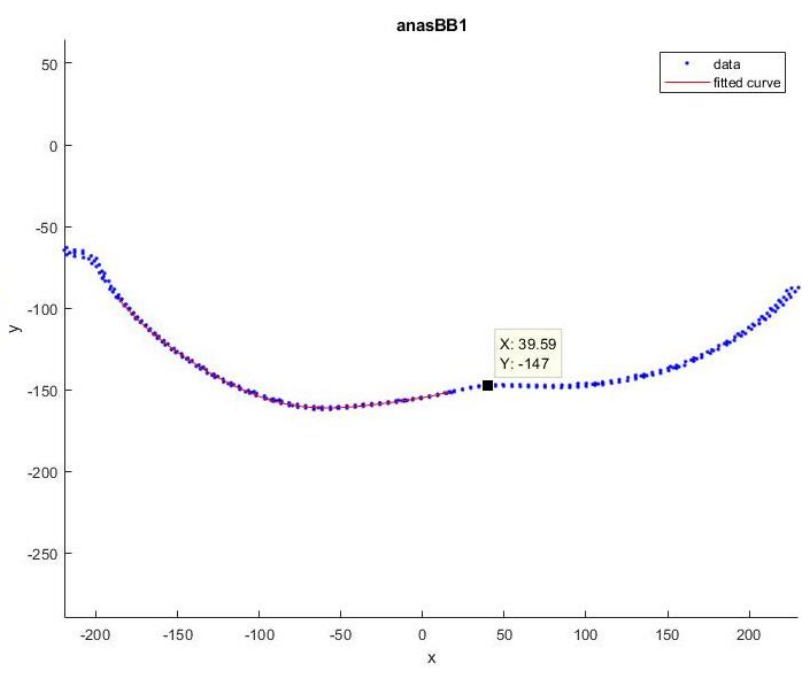

(j)

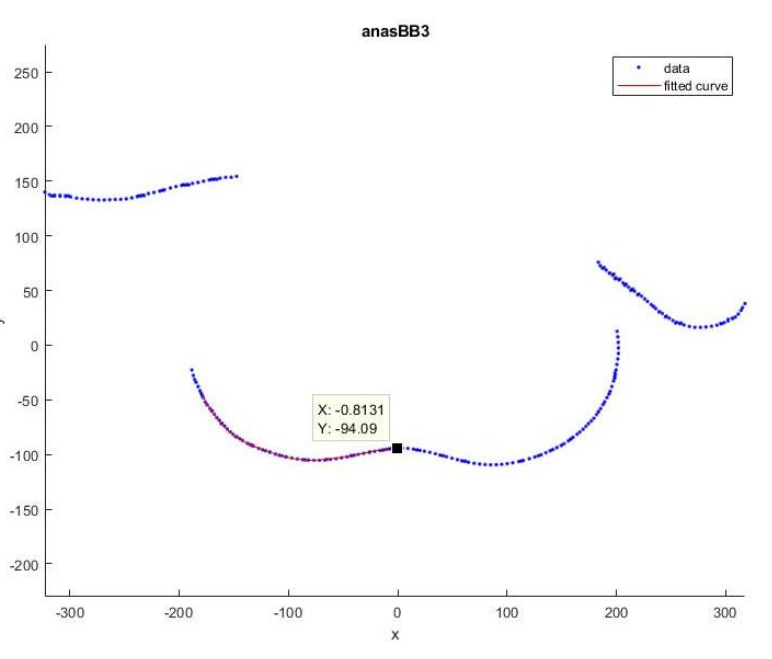

(I) 


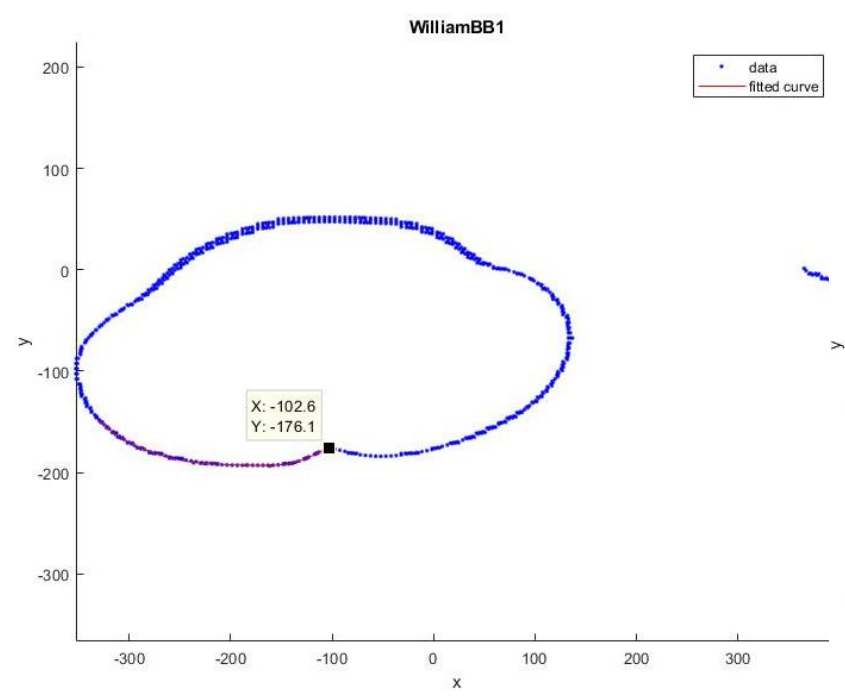

(m)

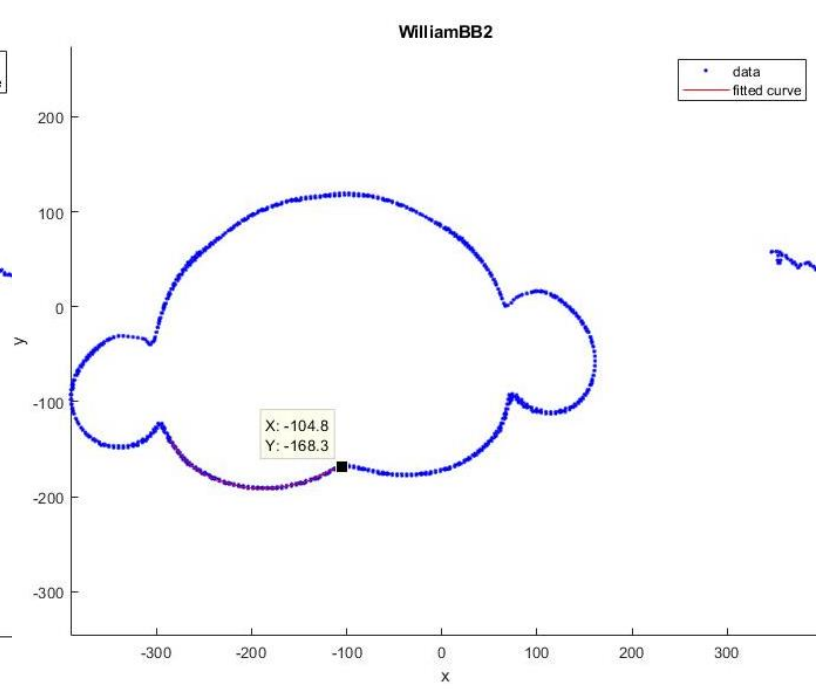

(n)

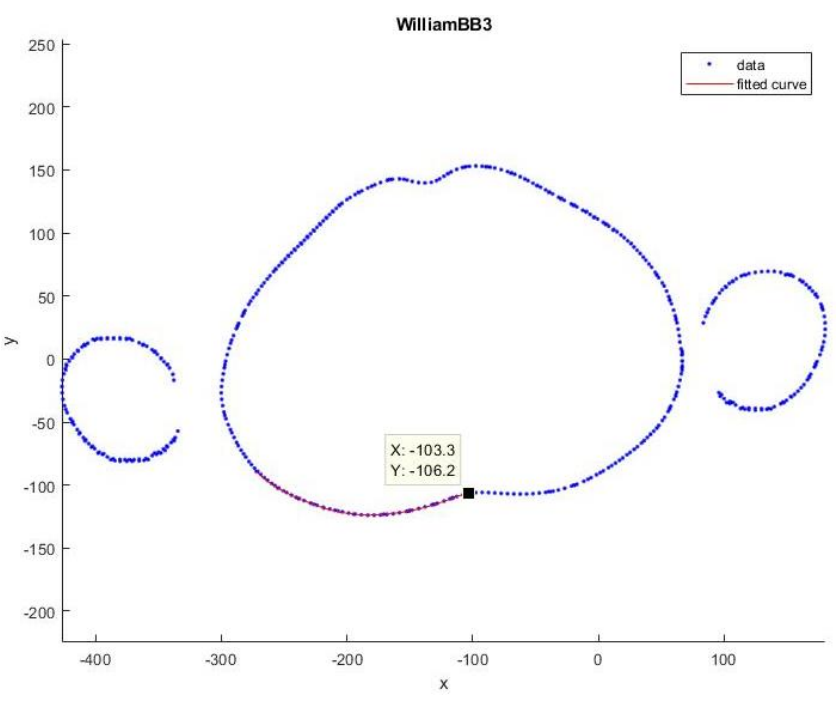

(o)

Figure 31. The back shape on the transverse plane of the body at the different level of lumbar, thoracic I and thoracic II. (a) thoracic I of subject 1 with BMI of 18.8 (b) thoracic II of subject 1 with BMI of 18.8 (c) lumbar of subject 1 with BMI of 18.8 (d) thoracic I of subject 2 with BMI of 20.1 (e) thoracic Il of subject 2 with BMI of 20.1 (f) lumbar of subject 2 with BMI of 20.1 (g) thoracic I of subject 3 with BMI of 22.9 (h) thoracic II of subject 3 with BMI of 22.9 (i) lumbar of subject 3 with BMI of 22.9 (j) thoracic I of subject 4 with BMI of 29.1 ( $\mathrm{k}$ ) thoracic Il of subject 4 with BMI of 29.1 (I) lumbar I of subject 4 with BMI of 29.1 (m) thoracic I of subject 5 with BMI of 31.9 (n) thoracic II of subject 5 with BMI of 31.9 (o) lumbar of subject 5 with BMI of 31.9 


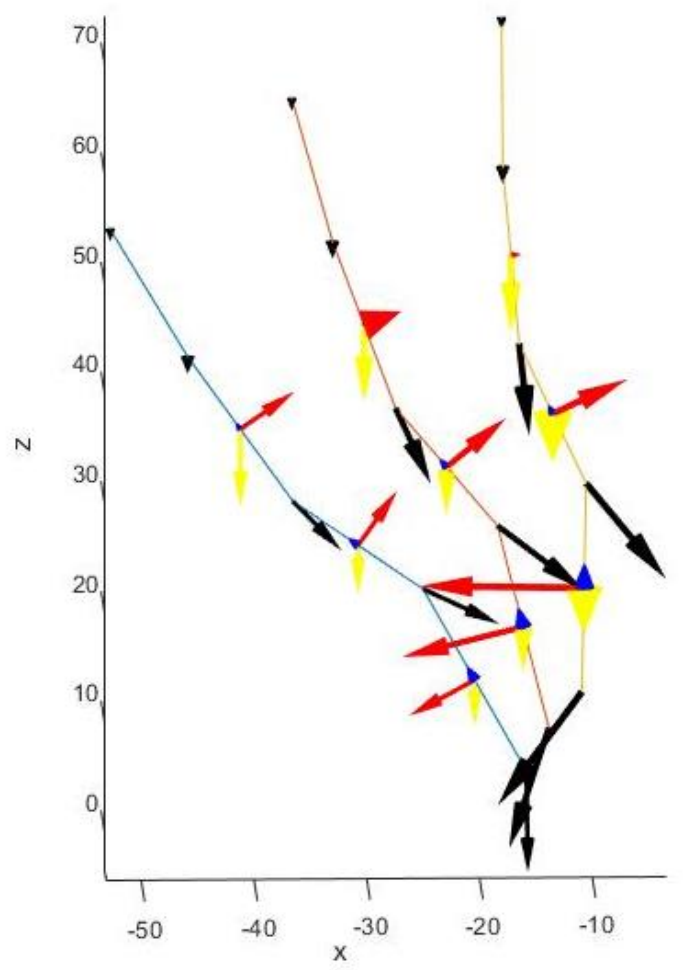

Figure 32. the force diagram of the erected spine when the backrest recline angle is 105 degree, 120 degree and 135 degree

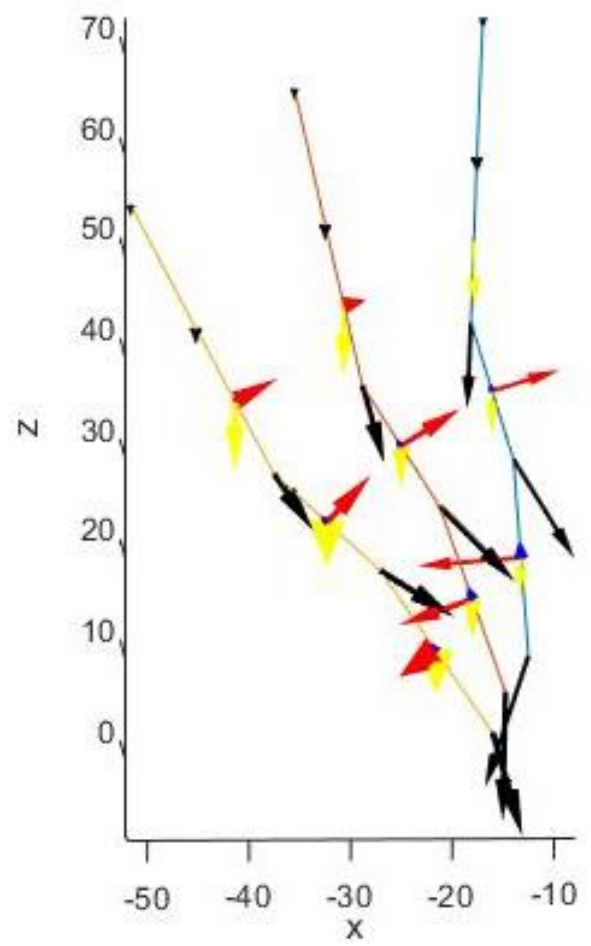

Figure 33. the force diagram of the normal spine when the backrest recline angle is 105 degree, 120 degree and 135 degree 


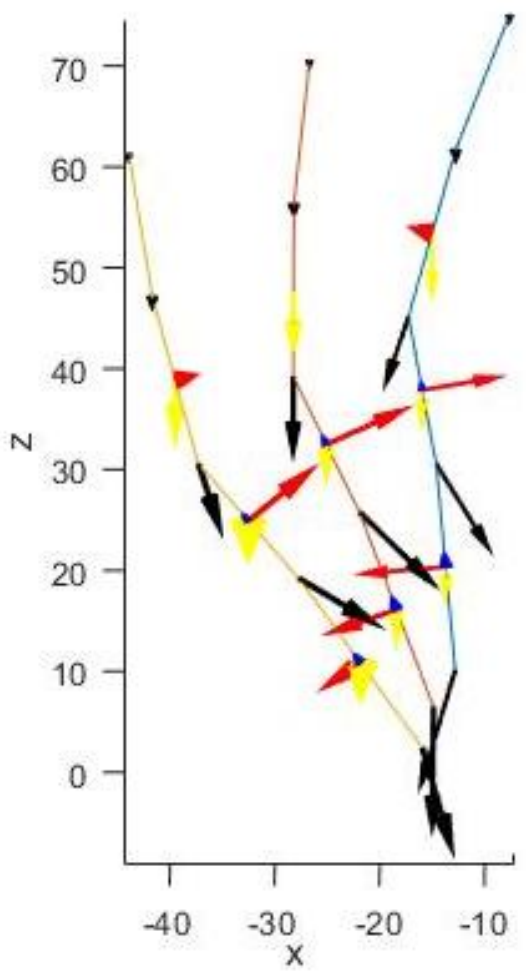

Figure 34. the force diagram of the slouched spine when the backrest recline angle is 105 degree, 120 degree and 135 degree 